\title{
STABLE FINITELY HOMOGENEOUS STRUCTURES
}

\author{
G. CHERLIN ${ }^{1}$ AND A. H. LACHLAN
}

\begin{abstract}
Let $L$ be a finite relational language and $\operatorname{Hom}(L, \omega)$ denote the class of countable $L$-structures which are stable and homogeneous. The main result of the paper is that there exists a natural number $c(L)$ such that for any transitive $\mathcal{M} \in \operatorname{Hom}(L ; \omega)$, if $E$ is a maximal 0 -definable equivalence relation on $\mathcal{M}$, then either $|\mathcal{M} / E|<c(L)$, or $\mathcal{M} / E$ is coordinatizable. In an earlier paper the second author analyzed certain subclasses $\operatorname{Hom}(L, r)(r<\omega)$ of $\operatorname{Hom}(L, \omega)$ for all sufficiently small $r$. Thus the earlier analysis now applies to $\operatorname{Hom}(L, \omega)$.
\end{abstract}

\section{Introduction.}

1.1. Statement of the main theorem. A relational system $\mathcal{M}$ is finitely homogeneous iff its theory admits elimination of quantifiers in a finite relational language $L$. For $\mathcal{M}$ countable, an equivalent condition (for a fixed language $L$ ) is given by

(*) Any isomorphism between two finite $L$-substructures of $\mathcal{M}$ is induced by some automorphism of $\mathcal{M}$.

A general theory of such structures is developed in [13]. This theory will be reviewed in more detail below, but the thrust of it may be summarized as follows. Let a finite relational language $L$ be specified, let $\operatorname{Hom}(L)$ be the class of $L$-structures $\mathcal{M}$ whose theories admit elimination of quantifiers (in the given language), and let $\operatorname{Hom}(L, \omega)$ denote the class of stable structures in $\operatorname{Hom}(L)$ (in the sense of [20]), which includes the class of finite structures in $\operatorname{Hom}(L)$.

Then the goal of the theory is to give a complete structural description of the structures in $\operatorname{Hom}(L, \omega)$ in terms of a finite number of numerical invariants, called dimensions. However the theory as presented in [13] accomplishes less than this, for the following technical reason.

Any structure $\mathcal{M}$ in $\operatorname{Hom}(L, \omega)$ has a "complete $\operatorname{rank} " \operatorname{rk}(\mathcal{M})$ which is a finite integer providing a rough measure of the complexity of $\mathcal{M}$. Let $\operatorname{Hom}(L, r)$ be $\{\mathcal{M} \in \operatorname{Hom}(L, \omega): \operatorname{rk}(M) \leq r\}$, so that $\operatorname{Hom}(L, \omega)=\bigcup_{r} \operatorname{Hom}(L, r)$. The structure theory given in $[\mathbf{1 3}]$ applies to each of the classes $\operatorname{Hom}(L, r)$, and so to complete the theory it is only necessary to prove

THEOREM 1. Given $L$ a finite relational language there is an integer $r$ for which $\operatorname{Hom}(L, \omega)=\operatorname{Hom}(L, r)$.

For binary languages $L$ this was proved in [14]. The general case will be treated here. We rely heavily on the theory of finite permutation groups in the manner

Received by the editors August 19, 1985.

1980 Mathematics Subject Classification. Primary 03C10, 20B99.

${ }^{1}$ First and second authors supported respectively by NSF Grants MCS81-02383 and MCS8301806, and NSERC Grant A3040. The bulk of this research was carried out while the first author was visiting Simon Fraser University (Fall 1982). 
of [5]. In this connection, we note that we are grateful to Chris Godsil, Brian Hartley, and Dugald Macpherson for discussing group-theoretic issues which arose in the course of the work. We also have a special debt to Bill Kantor who greatly simplified the proof of the existence of indiscernibles, see $\S 5$, as well as advising us on many other points.

1.2. Permutation structures. In order to present our results in the most natural way we need to adopt the point of view of permutation groups. A permutation structure is a pair $(M ; G)$ such that $M$ is a countable nonempty set and $G \leq \operatorname{Sym}(M)$ satisfies:

C1. $M^{n} / G$ is finite for each $n$.

C2. $G$ is complete in the topology of pointwise convergence.

Let $(M ; G),(N ; H)$ be permutation structures. We call $\varphi: M \rightarrow N$ an isomorphism of $(M ; G)$ and $(N ; H)$ if $\varphi$ is a bijection and $\varphi(G)=H$, where $\varphi(g)={ }_{\operatorname{dfn}} \varphi g \varphi^{-1} \in$ $\operatorname{Sym}(N)$ for each $g \in G$.

Let $\mathcal{M}$ be a countable $\aleph_{0}$-categorical relational structure with universe $M$. To $\mathcal{M}$ we associate the permutation structure $(M ; G)$ with $G=\operatorname{Aut}(\mathcal{M})$. By an abuse of notation we write $\mathcal{M}=(M ; G)$. Note that $\mathrm{C} 1$ is satisfied by the theorem of Engeler, Ryll-Nardzewski, and Svenonius, while C2 is immediate from the definition of automorphism for relational structures.

Let $(M ; G)$ be a permutation structure. The canonical language $L$ for $(M ; G)$ has $n$-ary relation symbols $R_{n, 1}, \ldots, R_{n, k_{n}}$, with $k_{n}=\left|M^{n} / G\right|$, for each $n, 1 \leq n<\omega$. Let $\mathcal{M}$ be an $L$-structure with $|\mathcal{M}|=M$ and

$$
\left\{\left(R_{n, i}\right)^{\mathcal{M}}: 1 \leq i \leq k_{n}\right\}=M^{n} / G \quad(1 \leq n<\omega) .
$$

Then $(M ; G)$ is the permutation structure associated to $\mathcal{M}$. Thus every permutation structure is associated to some relational structure.

Let $\mathcal{M}, \mathcal{N}$ be countable $\aleph_{0}$-categorical relational structures. If $\varphi:|\mathcal{M}| \rightarrow|\mathcal{N}|$ is an isomorphism of $\mathcal{M}$ and $\mathcal{N}$ as relational structures, then $\varphi$ is also an isomorphism of permutation structures. But the converse is false; the corresponding permutation structures may be isomorphic while $\mathcal{M}, \mathcal{N}$ have different languages.

If $H \leq \operatorname{Sym}(X), M \subseteq X$ is $H$-invariant, and $(M ; G)$ is the permutation structure with $G=\{h \mid M: h \in H\}$, then we shall sometimes abuse notation by writing $(M ; H)$ to mean $(M ; G)$. Also, the action of $G$ is understood to be extended in the obvious way to all sets definable over $M$.

Let $\mathcal{M}=(M ; G)$ be a permutation structure and $E$ be a $G$-invariant equivalence relation. The quotient $\mathcal{M} / E$ is $(M / E ; H)$ where $H \leq \operatorname{Sym}(M / E)$ is the group induced by $G$. According to our conventions we could write $(M / E ; G)$ for $(M / E ; H)$.

It is important to have a clear understanding of finite homogeneity in this context. A permutation structure $(M ; G)$ is called $k$-ary if the following holds for all $n$, in addition to $\mathrm{C} 1, \mathrm{C} 2$ :

$\mathrm{C} 3{ }_{k}$. Any $n$-tuples $\bar{a}, \bar{b} \in M^{n}$ such that each $k$-tuple $\bar{a}^{\prime} \subseteq \bar{a}$ is conjugate under $G$ to the corresponding $k$-tuple $\bar{b}^{\prime} \subseteq \bar{b}$ are themselves conjugate under $G$.

If $\mathrm{C} 3{ }_{k}$ holds, then $\mathrm{C} 1$ becomes: $M^{k} / G$ is finite. These are the permutation structures arising from structures in $\operatorname{Hom}(L)$, where $L$ is a finite relational language containing only $i$-ary relations for $i \leq k$.

For any structure $\mathcal{M}$ let $s_{n}(\mathcal{M})$ denote the number of $n$-types over $\varnothing$, which is $\left|M^{n} / G\right|$ if $\mathcal{M}=(M ; G)$. In practice, the study of $\operatorname{Hom}(L)$ for a fixed language $L$ 


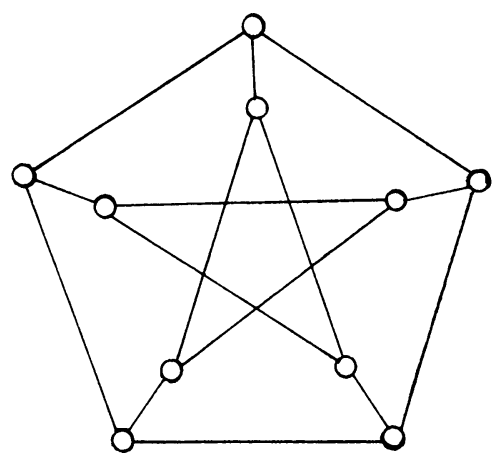

FIGURE 1

amounts to the study of structures $\mathcal{M}$ of arity at most $k$, with $s_{k}(\mathcal{M}) \leq s$, where $k$ and $s$ are fixed.

Notice that formation of quotients decreases the parameter $s_{n}(\mathcal{M})$ for each $n$. Unfortunately the arity $k$ may itself increase. For example, let $M$ consist of the nonzero vectors in $V(2, q)$, the 2-dimensional vector space over $F_{q}, G=\operatorname{SL}(2, q)$, and $u E v$ hold if $u=\lambda v$ for some $\lambda \in F_{q}$. Then $\mathcal{M}=(M ; G)$ is binary, but its natural quotient $\mathcal{M} / E$ is only 4 -ary. In fact, any finite transitive (meaning $s_{1}(\mathcal{M})=1$ ) permutation structure $\mathcal{M}$ is isomorphic to a quotient of a binary structure. If $\mathcal{M}=(M ; G)$, let $X$ be the set of elements of $G, a \in M$, and $G_{a}$ be the stabilizer of $a$ in $G$. Let $H=\left\{\pi_{g}: g \in G\right\} \leq \operatorname{Sym}(X)$, where $\pi_{g}(x)=g x(x \in X)$, and $x E y$ holds iff $x^{-1} y \in G_{a}$. Then $(X ; H)$ is a permutation structure and $(X ; H) / E \cong \mathcal{M}$.

1.3. Coordinatization. We will state a dichotomy theorem below from which Theorem 1 follows easily. It is necessary to consider first the notion of a coordinatizable structure. This notion is very close to that of perfect structure used in $[\mathbf{1 3}$, 14].

Let $(M ; G)$ be a permutation structure and $\mathcal{F}=\left\{H_{i}: i \in I\right\}$ be a family of pairwise disjoint subsets of $M$. $₹$ is mutually indiscernible if every $\pi \in \operatorname{Sym}(M)$ which fixes each $H_{i}$ setwise extends to a member of $G$. For an infinite set $X$ let $\operatorname{Alt}(X)$ mean the same as $\operatorname{Sym}(X)$. $\mp$ is mutually quasi-indiscernible if for every family $\left\{\pi_{i}: i \in I\right\}$ with $\pi_{i} \in \operatorname{Alt}\left(H_{i}\right)(i \in I)$ there exists $g \in G$ such that $g\left\lceil H_{i}=\pi_{i}\right.$ $(i \in I)$.

A permutation structure $(H ; G)$ is a (twisted) coordinate system if there is a $G$ invariant equivalence relation $E$ such that $H / E=\left\{H_{i}: i \in I\right\}$ is a finite mutually (quasi-) indiscernible family on which $G$ acts transitively, and $\left|H_{i}\right| \geq 5(i \in I)$. The degenerate case in which $G=\operatorname{Sym}(H)$ is allowed and even typical. The $H_{i}$ are the components of $H$.

Given a possibly twisted coordinate system $(H ; G)$ with components $H_{i}$ and $k$ such that $2 k \leq\left|H_{i}\right|$, define the Grassmannian structure:

$$
\operatorname{Gr}_{k}(H ; G)=\left(\left\{X \subseteq H: \forall i\left(\left|X \cap H_{i}\right|=k\right)\right\} ; G\right) .
$$

A structure $\mathcal{M}$ is coordinatizable if it is isomorphic (as a permutation structure) with a structure $\operatorname{Gr}_{k}(H ; G)$. One of the best known examples is the Petersen graph (Figure 1), which can be identified with

$$
\tau=\mathrm{Gr}_{2}(\{0,1,2,3,4\} ; \operatorname{Sym}(5)) .
$$


When $\mathcal{M}=(M ; G)$ is coordinatizable, it is quite easy to see that the coordinatization is essentially unique since $\operatorname{Soc}(G) \cong[\operatorname{Alt}(n)]^{d}$ where $d$ is the number of components and $n$ is their size.

1.4. A dichotomy. Most of our effort will be aimed at proving the following.

THEOREM 2 (DICHOTOMY THEOREM). Let a finite relational language $L$ be fixed. There is an integer $m$ such that for every $\mathcal{M} \in \operatorname{Hom}(L ; \omega)$ and every maximal 0 -definable equivalence relation $E$ on $\mathcal{M}$ one of the following occurs.

(A) $|M / E| \leq m$,

(B) $\mathcal{M} / E$ is quasi-coordinatizable.

This is related to the Coordinatization Theorem of [7]. The 0 -definable equivalence relations on $\mathcal{M}$ are the major source of difficulties, since $\mathcal{M} / E$ need no longer be in $\operatorname{Hom}(L)$ as noted earlier. If there are no 0 -definable equivalence relations on $\mathcal{M}$ we say that $\mathcal{M}$ is primitive, and then in the case of finite $\mathcal{M}$ the results of the past decade in primitive permutation groups are directly applicable.

In general for $\mathcal{M} \in \operatorname{Hom}(L)$ the set of 0 -definable equivalence relations on $\mathcal{M}$ forms a finite lattice, of size at most $\left|M^{2} / \operatorname{Aut}(\mathcal{M})\right|$, and we will refer to the height of this lattice as the height of $\mathcal{M}, \mathrm{ht}(\mathcal{M})$. The Dichotomy Theorem will be proved by induction on $h t(\mathcal{M})$.

1.5. Structure theory. We will now indicate the main consequence of Theorem 1 , which is described in detail in $[\mathbf{1 3}]$.

DEFINITION. 1. If $(H ; G)$ is a coordinate system with components $H_{i}(1 \leq i \leq d)$ and $\mathcal{M} \simeq \operatorname{Gr}_{k}(H ; G)$ then we define

$$
\begin{aligned}
\operatorname{dim}(\mathcal{M}) & \left.=\left|H_{i}\right| \quad \text { (independently of } i\right), \\
\operatorname{wd}(\mathcal{M}) & =d, \quad \operatorname{ind}(\mathcal{M})=k
\end{aligned}
$$

called the dimension, width, and index of $\mathcal{M}$ respectively.

2. Let $\mathcal{M}=(M ; G)$ and $E_{0}, E_{1}$ be $G$-invariant equivalence relations such that $\operatorname{Fld}\left(E_{0}\right)=\operatorname{Fld}\left(E_{1}\right) \subseteq M, E_{1}<E_{0}$, and $G$ acts transitively on $M / E_{0}$. Let $C \in$ $M / E_{0}$ and $C=\left(C / E_{1} ; K\right)$, where $K \leq \operatorname{Sym}\left(C / E_{1}\right)$ is the group induced by the setwise stabilizer of $C$ in $G$. Let

$$
C \cong \operatorname{Gr}_{k}(H ; G)
$$

for some coordinate system $(H ; G)$. Then set

$$
\left(E_{0} / E_{1}\right)-\operatorname{dim}(\mathcal{M})=\operatorname{dim}(\mathcal{C}) .
$$

$\left(E_{0} / E_{1}\right)-\operatorname{dim}(\mathcal{M})$ is only defined when $\operatorname{Fld}\left(E_{0}\right)=\operatorname{Fld}\left(E_{1}\right)$ and $E_{1}<E_{0}$. Further if $(*)$ holds then it is easy to recover $(H ; G)$ from $C$, as noted earlier, so the dimension is well defined.

3. The dimensions of $\mathcal{M}$ are the cardinals $\left(E_{0} / E_{1}\right)-\operatorname{dim}(\mathcal{M})$ when defined. If $\mathcal{M} \in \operatorname{Hom}(L)$, the dimensions are labeled by pairs of quantifier-free $L$-formulas $\left(\varphi_{0}\left(x_{0}, x_{1}\right), \varphi_{1}\left(x_{0}, x_{1}\right)\right)$ such that $\varphi_{i}$ defines $E_{i}(i<2)$.

Now it is proved in [13] that with $L$ and $r$ fixed:

Fact 1. Every structure $\mathcal{M}$ can be canonically "shrunk" to a structure $\mathcal{M}_{0} \in \operatorname{Hom}(L ; r)$, a homogeneous substructure of $\mathcal{M}$, all of whose dimensions are bounded.

Fact 2. The isomorphism type of $\mathcal{M}$ over $M_{0}$ is uniquely determined by $\mathcal{M}_{0}$ and the dimensions of $\mathcal{M}$. 
Fact 3. The size of $\mathcal{M}_{0}$, i.e., $\left|M_{0}\right|$, is bounded by a function of $L$ and $r$.

Fact 4. If the structures in $\operatorname{Hom}(L, r)$ are placed in "families" corresponding to the various structures $\mathcal{M}_{0}$, we obtain finitely many families, each of which is parametrized by a set of dimensions, which may vary freely above a certain minimum (if there are no dimensions, the family degenerates to a single structure $\mathcal{M}_{0}$, said to be sporadic for $L$ and $r$ ).

As stated earlier, the natural context in which to develop such a structure theory would seem to be $\operatorname{Hom}(L, \omega)$, and the restriction to $\operatorname{Hom}(L, r)$ comes about for technical reasons. Now Theorem 1 allows us to apply the theory to $\operatorname{Hom}(L, \omega)$ ex post facto.

1.6. The larger context. In all probability the classes $\operatorname{Hom}(L, \omega)$ are best viewed as special subclasses of the class of $\aleph_{0}$-categorical $\aleph_{0}$-stable structures, for which we have a general theory [7]. Theorem 1 is an analog of the theorem that such structures are of finite Morley rank, but the proof is quite different.

In [7] it is shown how to "coordinatize" infinite $\aleph_{0}$-categorical $\aleph_{0}$-stable structures using strongly minimal sets. This is related to $\S 1.3$, but whereas the strongly minimal sets arising in our context are degenerate (the algebraic closure of a set is the union of the algebraic closures of its elements), in the general case affine and projective geometries over finite fields are involved.

It was conjectured at one point that the class $\bigcup\{\operatorname{Hom}(L, \omega): L$ finite, relational $\}$ consisted exactly of the "disintegrated" structures (in which all coordinatizing strongly minimal sets are degenerated). The following simple example shows this is not so.

EXAMPLE. Let $S$ be a set, $G=\operatorname{Sym}(S)$, and let $M$ be the set of all structures $(X ; E)$ where

$$
\begin{aligned}
& X \subseteq S \text { has four elements, } \\
& E \text { is an equivalence relation on } X \text { with two classes } \\
& \text { having two elements each. }
\end{aligned}
$$

Then $\mathcal{M}=(M ; G)$ is a permutation structure under the natural action of $G$ on $M$, and may be viewed as a relational system $(\S 1.2)$.

$\mathcal{M}$ is not $k$-ary for any value of $k$. Let $a_{1}, \ldots, a_{k+1}, b_{1}, \ldots, b_{k+1} \in S$ be distinct, $X_{i}=\left\{a_{i}, a_{i+1}, b_{i}, b_{i+1}\right\}$ for $1 \leq i \leq k, X_{k+1}=\left\{a_{1}, a_{k+1}, b_{1}, b_{k+1}\right\}$, and let $E_{i}$ on $X_{i}$ split the $a$ 's apart from the $b$ 's, while $E_{k+1}^{\prime}$ splits $a_{1}, b_{k+1}$ apart from $b_{1}, a_{k+1}$. Let $m_{i}=\left(X_{i}, E_{i}\right), m_{k+1}^{\prime}=\left(X_{k+1}, E_{k+1}^{\prime}\right)$. Then $k$-arity is refuted by comparing $m_{1}, \ldots, m_{k+1}$ and $m_{1}, \ldots, m_{k}, m_{k+1}^{\prime}$.

Thus the proper generalization of $[\mathbf{1 3}]$ to the class of disintegrated structures is not known as yet.

1.7. Finitely homogeneous structures in general. The study of finitely homogeneous structures in general, with no auxiliary hypothesis of finiteness or stability, amounts to the direct investigation of amalgamation classes: families $A$ of finite structures, closed under substructure and isomorphism, and satisfying the amalgamation property. From this point of view the fundamental problem is the study of the entailment relation

$$
\bigwedge_{I} A_{i} \Rightarrow \bigvee_{J} B_{j}
$$


defined as follows: any amalgamation class $A$ containing all the $\mathcal{A}_{i}$ must contain also some $B_{j}$. We are interested in the case in which $I, J$ are finite-and primarily in the case in which $J$ is a singleton. It is easy to see that the problem is essentially unchanged if we restrict $I$ to be a singleton. Then the fundamental problem, in abstract form, is this:

Is the entailment relation decidable?

The results in this paper imply that a restricted problem, in which the amalgamation classes are taken to be finite (modulo isomorphisms), is decidable. The other half of the problem, in which the amalgamation classes are assumed infinite, has at least one special feature: Ramsey's theorem applies. In certain very special cases, this is enough to settle the entailment problem positively. Specifically, if one studies structures which are homogeneous for a binary language in which only two 2-types are realized, then there are only countably many, all easily describable $[\mathbf{1 2}$, 17].

A classification at the next level-binary structures with three 2-types-would probably cast significantly more light on the general case, because as is well known there are $2^{\aleph_{0}}$ inequivalent examples at this stage.

2. Preliminaries to the proof of Theorem 2 and reduction of Theorem 1 to Theorem 2. We will restate Theorem 2 in the form most suited to its proof. Two crucial lemmas, Lemmas A and B, will be stated. Lemma A will be proved here. The proof of Lemma B is deferred until $\S 5$ because of its complexity. Finally, it is shown that Theorem 1 follows from Theorem 2 .

2.1. Theorem 2: Sketch. Theorem 2 will be proved in the following formulation.

THEOREM 2. Given a fixed finite relational language $L$ and an integer $h$, there is an integer $c(h)$ such that:

(*) For any transitive $\mathcal{M} \in \operatorname{Hom}(L, \omega)$ of height at most $h$, and any maximal 0-definable equivalence relation $E$ on $\mathcal{M}$, either:

1. $|M / E|<c(h)$, or

2. $\mathcal{M} / E$ is coordinatizable.

Here the height is one less than the length of the longest chain of 0 -definable equivalence relations on $M$. In particular a structure of height 0 contains a unique element, and $(*)$ is vacuous.

Observe that the height $h$ of all structures in $\operatorname{Hom}(L)$ is uniformly bounded, given $L$; indeed the lattice of 0 -definable equivalence relations is finite of bounded size. Hence the original version of Theorem 2 is equivalent to the present formulation.

The proof will proceed by induction on the height $h$, primarily by a grouptheoretic argument, but with the help of the following very convenient lemma.

Terminology. Let $\mathcal{F}, \mathcal{F}^{\prime}$ be families of disjoint subsets of a structure $\mathcal{M}=(M ; G)$. $\mathcal{F}^{\prime}$ is a refinement of $\mathcal{F}$ if there is a bijection $X \leftrightarrow X^{\prime}$ between $\mathcal{F}$ and $\mathcal{F}^{\prime}$ such that $X^{\prime} \subseteq X$ for all $X \in \mathcal{F}$.

$\mathcal{F}^{\prime}$ is a replacement for $\mathcal{F}$ in $\mathcal{M}$ if $\mathcal{F}^{\prime}$ is a refinement of $\mathcal{F}$ such that every permutation of $₹$ induced by $g \in G$ is also induced by some $g^{\prime} \in G$ leaving $\mathcal{F}^{\prime}$ invariant.

Lemma A (Replacement Lemma). Assume (*) of Theorem 2 holds for $h$. Let $F: \omega \rightarrow \omega$ be given. Then there is $f \in \omega$ such that for all transitive $\mathcal{M} \in \operatorname{Hom}(L)$ 
of height at most $h+1$, for any 0 -definable equivalence relation $E$ on $M$ there is $e \leq f$ with

(**) Any $\mp \subseteq M / E$ of cardinality $F(e)$ has a replacement $\mathcal{F}^{\prime}$ in $\mathcal{M}$ by sets of size $<e$.

We view $\mathcal{M}$ as a permutation structure $(M ; G)$, where $G$ is the automorphism group of the relational structure, and fix a maximal 0-definable equivalence relation $E$. By an application of 7.4 of [7], in carrying out the induction step of the proof of Theorem 2 we may assume that $M$ is finite. There is a quotient structure $(M / E ; H)$ which is primitive since $E$ is maximal. When $\operatorname{Soc}(H)$ is abelian, besides the Replacement Lemma we shall need

LEMMA B. There is a function $m(s, n)$ such that for any transitive permutation structure $(M ; G)$ satisfying $\left|M^{5} / G\right| \leq s,|M| \geq m(s, n)$ there is a set $I \subseteq M$ of $n$ indiscernibles. (That is, $G$ induces $\operatorname{Sym}(I)$ on $I$.)

This result does not appear to be directly derivable from anything in the literature, though it slightly resembles the result of $[\mathbf{3}]$.

2.2. Proof of Lemma A. Throughout this section we suppose that $L$ is a fixed finite relational language. All of the numerical bounds established here depend implicitly on $L$. Before proving the Replacement Lemma we establish some facts that will be needed in the proof.

Let $\mathcal{M}=(M ; G)$ be a permutation structure. A structure $\mathcal{N}=(N ; H)$ is called a section of $\mathcal{M}$ if there exist $G$-invariant equivalence relations $E_{0}>E_{1}$ on $M$ and an $E_{0}$-class $C$ such that $N=C / E_{1}$ and $H \leq \operatorname{Sym}(N)$ is the group of permutations induced by $G(\{C\})$, the set-stabilizer of $C$ in $G$.

When we refer to Grassmannians in this section we have in mind the more general case in which the coordinate system may be twisted.

LEMMA 1. There exist $w_{L}, i_{L} \in \omega$, and a function $F_{L}: \omega \rightarrow \omega$ such that, if $\mathcal{N}$ is a section of $\mathcal{M} \in \operatorname{Hom}(L)$ which is coordinatizable, then $\operatorname{wd}(\mathcal{N}) \leq w_{L}$, $\operatorname{ind}(\mathcal{N}) \leq i_{L}$, and $|\mathcal{N}| \leq F_{L}(\operatorname{dim}(\mathcal{N}))$ if $\operatorname{dim}(\mathcal{N})$ is finite.

Proof. Suppose that $(H ; G)$ is a possibly twisted coordinate system for $\mathcal{N}$ with components $H_{i}(1 \leq i \leq d)$ and $\mathcal{N} \simeq \mathrm{Gr}_{k}(H ; G)$. By definition $2 k \leq \operatorname{dim}(\mathcal{N})$, $\operatorname{dim}(\mathcal{N})$ being the common cardinality of the sets $H_{i}$. Then $\operatorname{ind}(\mathcal{N})=k$ and $\operatorname{wd}(\mathcal{N})=d$ by definition. For $X, Y \in \mathrm{Gr}_{k}(H ; G),|X \cap Y|$ can take any value $\leq k d$, and this number is invariant if $X, Y$ are moved by $g \in G$. Thus $s_{2}(\mathcal{N}) \geq k d+1$. Moreover, $s_{2}(\mathcal{N}) \leq s_{2}(\mathcal{M})$ since $\mathcal{N}$ is a section of $\mathcal{M}, s_{2}(\mathcal{M})$ is bounded in terms of $L$, and $k, d \geq 1$. Therefore we can take both $w_{L}$ and $i_{L}$ to be the bound that $L$ imposes on $s_{2}(\mathcal{M})$ for $\mathcal{M} \in \operatorname{Hom}(L)$. Finally, from the definition of Grassmannian,

$$
|\mathcal{N}|=\left(\begin{array}{c}
\operatorname{dim}(\mathcal{N}) \\
k
\end{array}\right)^{d}
$$

which establishes the required bound on $|\mathcal{N}|$.

Our other preliminaries are concerned with quasi-indiscernible sets. Let $\mathcal{M}=$ $(M ; G)$ be a permutation structure. We assume that $M$ is chosen such that $M^{m} \cap M^{n}=\varnothing$ for all $m<n<\omega$. We form a structure $\mathcal{M}^{\mathrm{eq}}=\left(M^{\mathrm{eq}} ; G\right)$ by 
letting

$$
\begin{aligned}
M^{\mathrm{eq}}=\{a:(\exists n<\omega)(\exists E)(E \text { is a } 0 \text {-definable } \\
\left.\left.\quad \text { equivalence relation on } M^{n} \text { and } a \in M^{n} / E\right)\right\}
\end{aligned}
$$

and letting $G$ act naturally on $M^{\text {eq }}$. By convention 1-tuples are identified with elements of $M$. Thus $M \subseteq M^{\mathrm{eq}}$ and $M$ is 0 -definable in $\mathcal{M}^{\mathrm{eq}}$. For $a \in M^{\mathrm{eq}}$, by $[a]$ we mean the least $m<\omega$ such that $\{a\}$ is $A$-definable in $\mathcal{M}^{\text {eq }}$ for some $A \subseteq M$ with $|A|=m$. For $X \subseteq M^{\text {eq }}$, by $[X]$ we mean $\sup \{[a]: a \in X\}$. We resolve the ambiguity in $[X]$ by viewing $X$ as a subset of $M^{\text {eq }}$ when we have both $X \subseteq M^{\text {eq }}$ and $X \in M^{\text {eq }}$.

Since in this paper we are concerned only with the case in which $\mathcal{M}$ is countable and atomic we define the notion of indiscernible set in a way which is convenient for our purpose but which would not agree with the usual model-theoretic definition in a wider context. A finite set $I \subseteq M^{\text {eq }}$ is indiscernible (quasi-indiscernible) in $\mathcal{M}$ if every (even) permutation of $I$ is induced by some element of $G$. An infinite set $I \subseteq M^{\mathrm{eq}}$ is indiscernible or quasi-indiscernible in $\mathcal{M}$ if every finite subset of $I$ is indiscernible.

For $X \subseteq M^{\text {eq }}$ let $G(X)$ denote the pointwise stabilizer of $X$ and $G(\{X\})$ its setwise stabilizer. One of the consequences of the classification of finite simple groups, see for example [5, Theorem 5.3], is

LEMMA 2. If $\mathcal{M}=(M ; G)$ is finite and sufficiently large, and $G$ acts 4-transitively, then $G \geq \operatorname{Alt}(M)$.

The next lemma describes the way in which invariant quasi-indiscernible sets can interact with each other. Recall the notion of mutual quasi-indiscernibility from $\S 1.3$.

LEMMA 3. Let $\mathcal{M}$ be a countable atomic structure and $I=\left\{I_{i}: i<n\right\}$ be a family of distinct 0-definable quasi-indiscernible subsets of $M^{\mathrm{eq}}$ such that $7 \leq$ $\left|I_{i}\right|<\aleph_{0}(i<n)$. There is an equivalence relation $E$ on $n$ such that

(i) for $i, j<n, i E j$ iff there is a 0 -definable bijection between $I_{i}$ and $I_{j}$, and

(ii) if $J \subseteq n$ is a set of representatives of distinct $E$-classes, then $J=\left\{I_{j}: j \in J\right\}$ is mutually quasi-indiscernible.

REMARK. If we assume $\mathcal{M}$ stable we can allow $\left|I_{i}\right| \leq \aleph_{0}$. This case will not be needed here so we ignore it.

PROOF. It is easy to adapt the proofs of Lemmas 7.6 and 7.8 of $[\mathbf{1 3}]$ to give a proof of the present result. Those lemmas are not directly applicable because they were formulated for indiscernible sets.

One of the key concepts in what follows is that of the closure of a subset $A$ of $M^{\text {eq }}$ in a quasi-indiscernible set $I \subseteq M^{\text {eq }}$. Define $I-\operatorname{cl}_{\mathcal{M}}(A)$ to be the least finite $B \subseteq I$ if any such that $2|B|<|I|$ and such that $I-B$ is quasi-indiscernible in $\mathcal{N}=\left(M^{\mathrm{eq}} ; G(A \cup B)\right)$. It is easy to check that, if such $B$ exists, then the family of all such $B$ is closed under intersection. The subscript $\mathcal{M}$ will be omitted when it is clear which structure we have in mind.

LEMMA 4. There exist $F: \omega^{3} \rightarrow \omega$ and $K: \omega^{2} \rightarrow \omega$ such that if $\mathcal{M} \in \operatorname{Hom}(L)$, $I \subseteq M^{\text {eq }}$ is quasi-indiscernible, $A, B \subseteq M$ are finite, $I$ is $B$-definable, and $F(|A|,|B|,[I])<|I|<\aleph_{0}$, then $I-\operatorname{cl}(A)$ exists and $|I-\operatorname{cl}(A)|<K(|A|,|B|)$. 
REMARK. If we assume that $\mathcal{M}$ is stable, then the conclusion holds also when $|I|=\aleph_{0}$. Comparing this lemma with the results in $[13, \S 7]$ we see that the notion of closure is better behaved with respect to quasi-indiscernibility than it is with respect to indiscernibility. To prove the corresponding lemma for indiscernibility we have to assume a bound on $\operatorname{rk}(\mathcal{M})$.

ProOF. Let $\mathcal{M}=(M ; G)$ be regarded as a permutation structure. Supposing that $|I|$ is large enough compared with $|A|,|B|$, and $[I]$, we shall show that $I-\operatorname{cl}(A)$ exists. Suppose $J \subseteq I$ is $G(A \cup B)$-invariant and $2|J| \leq|I|$. Then $|J|<s_{2 k}(\mathcal{M})$, where $k=|A|+|B|$, because for each $i \leq|J|$ there exists $J^{\prime} \subseteq I$ conjugate to $J$ under $G$ such that $\left|J \cap J^{\prime}\right|=i$. Since $|I|$ is large compared with $k$, there is a unique large orbit of $G(A \cup B)$ on $I$. Let $J$ denote the union of the remaining orbits. From above, $|J|<s_{2 k}(\mathcal{M})$. Since $L$ is fixed and $\mathcal{M} \in \operatorname{Hom}(L),|J|$ is bounded in terms of $k$.

ClaIM 1. $G(A \cup B \cup J)$ is transitive on $I-J$.

PROOF. By an argument similar to the one made above $G(A \cup B \cup J)$ has one large orbit on $I$ and the union $X$ of the remaining orbits is small. Since $X$ is $(A \cup B)$-definable and small, $X \subseteq J$ and the claim is proved.

ClaIM 2. Either $I-\operatorname{cl}(A)$ exists included in $J$, or there exist $C \subseteq I-J$ and $c \in I-(J \cup C)$ such that $|C| \leq 2$ and $G(A \cup B \cup J \cup C \cup\{c\})$ is not transitive on $I-(J \cup C \cup\{c\})$.

Proof. Suppose no such $C, c$ exist. Then $G(A \cup B \cup J)$ acts 4-transitively on $I-J$. By Lemma 2, since $I-J$ is large, $G(A \cup B \cup J)$ induces at least the alternating group on $I-J$ and so we are done.

For the rest suppose $C, c$ are as in Claim 2 with $|C|$ least possible. Repeating a previous argument we see that $H=G(A \cup B \cup J \cup C \cup\{c\})$ has one large orbit on $I$ and that the union of the remaining orbits is small with cardinality bounded in terms of $k$. From the choice of $C$ and $c$ there exists $d \in I-(J \cup C \cup\{c\})$ such that $d$ is in a small orbit of $H$. Let $R \subseteq I^{2}$ be the orbit of $G(A \cup B \cup J \cup C)$ containing $(c, d)$. Note that $R$ is $(A \cup B \cup J \cup C)$-definable and determines a possibly directed graph $\Gamma$ with vertex set $I-(J \cup C)$ and valency bounded in terms of $k$. The size of the connected components of $\Gamma$ can be bounded in terms of $k$; otherwise there would be too many binary relations $(A \cup B \cup J \cup C)$-definable on $I-(J \cup C)$. Thus there is an $(A \cup B \cup J \cup C)$-definable equivalence relation $S$ on $I-(J \cup C)$, whose classes are the connected components of $\Gamma$ and hence of size bounded in terms of $k$. Since $|C|$ is least possible, $G(A \cup B \cup J \cup C)$ is transitive on $I-(J \cup C)$. Hence all $S$-classes have the same size.

Let $D \subseteq M$ be such that $\{b\}$ is $D$-definable for all $b \in A \cup B \cup J \cup C$ and $|D|$ is least possible. We can bound $l=|D|$ in terms of $k$ and $[I]$. Let $m=|(I-(J \cup C)) / S|$. Since the $S$-classes are small, $m$ is very large compared with $k$ and $[I]$. Since $I$ is quasi-indiscernible, for any $i \leq m$ there exists $\left(D^{\prime}, S^{\prime}\right)$ conjugate to $(D, S)$ under $G$ such that the equivalence relations $S, S^{\prime}$ have exactly $i$ classes in common. Therefore $s_{2 l}(\mathcal{M})$ is very large. This contradicts the bound imposed by $L$ on $s_{2 l}(\mathcal{M})$. Hence $C$ and $c$ do not exist which means that $I-\operatorname{cl}(A)$ exists and is included in $J$.

Once $L$ is given we can easily trace through the argument to compute a suitable function $F$. To get the function $K$ we need only rcall that $|J|$ is bounded in terms of $k=|A|+|B|$. 
We are now ready to prove Lemma $A$. Recall that, when $\mathcal{F}^{\prime} \mathcal{F}^{\prime}$ are families of disjoint subsets of $M, \mathcal{F}^{\prime}$ is a replacement for $₹$ in $\mathcal{M}=(M ; G)$ iff $\mathcal{F}^{\prime}$ is a refinement of $₹$ such that every permutation of $₹$ induced by an element of $G$ is induced by some $g \in G$ leaving $\mathcal{F}^{\prime}$ invariant.

Lemma A [Replacement Lemma]. Assume $(*)$ of Theorem 2 holds for $h$. Let $F: \omega \rightarrow \omega$ be given. Then there is $f \in \omega$ such that for all finite transitive $\mathcal{M} \in \operatorname{Hom}(L)$ of height at most $h+1$, for any 0 -definable equivalence relation $E$ on $M$ there is $e \leq f$ such that

(**) Any $\mathcal{\exists} \subseteq M / E$ of cardinality $F(e)$ has a replacement $\mathcal{F}^{\prime}$ in $\mathcal{M}$ by sets of size $<e$.

The lemma is also true for infinite $\mathcal{M} \in \operatorname{Hom}(L, \omega)$. We need only the case in which $\mathcal{M}$ is finite.

PROOF. Recall that the dimensions of $\mathcal{M}$ are the dimensions of the coordinatizable sections of $\mathcal{M}$. Notice that it suffices to prove:

Proposition. Assume (*) of Theorem 2 holds for $h$. Let $F: \omega \rightarrow \omega$ be given. There exists $H: \omega \rightarrow \omega$ such that $H(j)>j(j \in \omega)$ and whenever $\mathcal{M}=(M ; G) \epsilon$ $\operatorname{Hom}(L)$ is finite and transitive with $\mathrm{ht}(\mathcal{M}) \leq h+1, E$ is a proper 0-definable equivalence relation on $M, C=\left(C ; G^{C}\right)$ is the structure induced on an $E$-class $C$ by its stabilizer $G(\{C\})$, and $i \in \omega$ is fixed so that

$$
\text { no dimension of } C \text { lies between } i \text { and } H(i) \text {, }
$$

then there exists $e$ with $i \leq e \leq H(i)$ such that

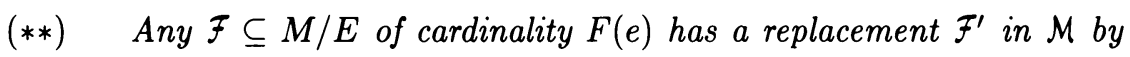
sets of size $<e$.

Suppose $H: \omega \rightarrow \omega$ satisfies the conclusion of the Proposition. Let $h_{0}=0$ and $h_{j+1}=H\left(h_{j}\right)(j \in \omega)$. From $L$ we can compute $k$ such that $\mathcal{M}$ and hence $C$ has less than $k$ dimensions. One of the intervals $\left(h_{j}, h_{j+1}\right)(j<k)$ contains no dimension of $C$. Hence there exists $e \leq h_{k}$ satsifying $(* *)$. Thus the conclusion of the lemma is satisfied by taking $f=h_{k}$. The rest of this section is devoted to the proof of the Proposition.

Let $\mathcal{M}, E, C$, and $C$ be as in the statement of the Proposition. If $E^{\prime}$ is a 0 definable equivalence relation on $M$ and $k^{\prime} \in \omega$, by a $\left(k^{\prime}, E^{\prime}\right)$-system of $\mathcal{M}$ we mean a family of pairwise disjoint, pairwise $G$-conjugate subsets of $M$, each member of which is a union of $k^{\prime}$ distinct $E^{\prime}$-classes.

Let $i \in \omega$ and $\mathcal{F}$ be a $(1, E)$-system of $\mathcal{M}$, i.e., a finite subset of $M / E$. Let $E=$ $E_{0}>E_{1}>\cdots>E_{j}$ be a maximal descending sequence of 0 -definable equivalence relations on $M$. Let $i \in \omega$ be such that any dimension of $C$ which is $>i$ is very large compared with $i$ and $|\mathcal{F}|$. We show how to find for each $l \leq j$ a positive integer $k_{l}$ and a $\left(k_{l}, E_{l}\right)$-system $\mathcal{F}_{l}$ of $\mathcal{M}$ such that

(1) $k_{0}=1, \mathcal{F}_{0}=\mathcal{F}$

(2) $\mathcal{F}_{l+1}$ is a replacement for $\mathcal{F}_{l}$ in $\mathcal{M}(l<j)$,

(3) $k_{l}$ is bounded terms of $i(l \leq j)$.

Since $E_{j}$ is the identity we take $e=k_{j}+1$. From the bound on $k_{j}$ we obtain a bound on $F(e)$ in terms of $i$. Finally, assuming $|\mathcal{F}| \leq F(e)$ we can compute $H(i)$ 
such that $C$ having no dimensions between $i$ and $H(i)$ is enough for the argument which bounds the $k_{l}$ in terms of $i$. The only point which remains to be explained is how we pass from $k_{l}$ and $\mathcal{F}_{l}$ to $k_{l+1}$ and $\mathcal{F}_{l+1}$ so as to satisfy (2) and (3). Since $j<h$, for (3) it is enough to bound $k_{l+1}$ in terms of $k_{l}$ and $i$.

For $l<j$ let $C_{l}$ be an $E_{l}$-class and $\mathcal{N}_{l}=\left(C_{l} / E_{l+1} ; G_{l}\right)$ be the structure induced on $C_{l} / E_{l+1}$ by $G\left(\left\{C_{l}\right\}\right)$. In passing from $\left(k_{l}, \mathcal{F}_{l}\right)$ to $\left(k_{l+1}, \mathcal{F}_{l+1}\right)$ there are three cases. Consider first the case in which $\mathcal{N}_{l}$ is not coordinatizable. Applying Theorem 2 for $h$ to the structure induced by $G\left(\left\{C_{l}\right\}\right)$ on $C_{l}$, we see that $\left|\mathcal{N}_{l}\right| \leq c(h)$, i.e., $E_{l+1}$ partitions each $E_{l}$-class into at most $c(h)$ pieces. Thus we can take $\mathcal{F}_{l+1}=\mathcal{F}_{l}$ and $k_{l+1}=k_{l}\left|\mathcal{N}_{l}\right|$. We have $k_{l+1}$ bounded in terms of $k_{l}$ as required. Consider next the case in which $\mathcal{N}_{l}$ is coordinatizable with dimension $\leq i$. From Lemma $1,\left|\mathcal{N}_{l}\right|$ is bounded in terms of $i$. Thus we take $\mathcal{F}_{l+1}=\mathcal{F}_{l}$ and $k_{l+1}=k_{l}\left|\mathcal{N}_{l}\right|$ as before.

Finally, consider the case in which $\mathcal{N}_{l}$ is coordinatizable with dimension which is very large compared with $i$ and $|\mathcal{F}|$. For each $E_{l}$-class $D$ there is a twisted coordinate system $\left(H_{D} ; G_{D}\right)$ attached to $D$ since the structure induced on $D$ by $G(\{D\})$ is quasi-coordinatizable. For $d \in D$ let $\operatorname{crd}(d) \subseteq H_{D}$ be the set corresponding to $d$ under the coordinatization. Any $a \in H_{D}$ can be identified with the set of all $\left(d_{0}, d_{1}\right) \in D^{2}$ such that $\operatorname{crd}\left(d_{0}\right) \cap \operatorname{crd}\left(d_{1}\right)=\{a\}$. Thus $H_{D}$ can be seen as a subset of $M^{\text {eq }}$ which is definable in $M^{\text {eq }}$ from $D$ regarded as an element of $M^{\text {eq }}$. Further, the components of $H_{D}$ are classes of a 0 -definable equivalence relation. Let $H_{D}$ denote the set of components of $H_{D}$, and let

$$
\not=\bigcup\left\{\not_{D}: D \in M / E_{l} \& \exists X\left(X \in \mathcal{F}_{l} \& D \subseteq X\right)\right\} .
$$

Let $\mathcal{M}^{\prime}=(M ; G(\not{H}))$, where $G(\mathcal{H})$ denotes the pointwise stabilizer of $\mathscr{H}$.

Since $\left|\nvdash_{D}\right|=\operatorname{wd}\left(\mathcal{N}_{l}\right) \leq w_{L}$ by Lemma 1 , there exists $A \subseteq M$ with $|A|$ bounded in terms of $k_{l}$ and $|\mathcal{F}|$ which fixes $\mathcal{F}$ pointwise. Moreover, if $I \in \nVdash$, then $|I|=\operatorname{dim}\left(\mathcal{N}_{l}\right)$ is large compared with $i$ and $|\mathcal{F}|$, and hence large compared with $|A|$. Note that members of $\mathcal{H}$ can be regarded as elements of $M^{\text {eq }}$ in a natural way and so $I-\operatorname{cl}_{\mathcal{M}}(\mathcal{H})$ makes sense for any indiscernible $I \subseteq M^{\text {eq }}$. By Lemma 4, for each $I \in \mathscr{H}, I-\operatorname{cl}_{\mathcal{M}}(\not{H})$ exists and is bounded in terms of $|\mathcal{F}|$ and $i$. Let $\mathcal{H}^{\prime}=\left\{I \backslash I-\operatorname{cl}_{\mathcal{M}}(\mathcal{H}): I \in \mathcal{H}\right\}$ and $\mathscr{H}_{D}^{\prime}=\left\{I \backslash I-\operatorname{cl}_{\mathcal{M}}(\mathscr{H}): I \in \mathscr{H}_{D}\right\}$ for each $D \in M / E_{l}$. $\mathscr{H}^{\prime}$ is a family of subsets of $M^{\text {eq }}$ which are 0-definable and quasi-indiscernible in the sense of $\mathcal{M}^{\prime}$. Let $\mathfrak{H}^{\prime \prime} \subseteq \mathcal{H}^{\prime}$ be maximal such that no two distinct members of $\mathcal{H}^{\prime \prime}$ have a 0-definable bijection between them. From Lemma $3 \mathcal{H}^{\prime \prime}$ is mutually quasi-indiscernible. Let $d=\operatorname{ind}\left(\mathcal{N}_{l}\right)$. Then an element of $D$ has $d$ coordinates in each memb er of $\varkappa_{D}$.

For each $I \in \mathcal{H}^{\prime \prime}$ choose $J(I) \subseteq I$ of cardinality $d$. For $I \in \mathscr{H}^{\prime}$ let $I^{\prime}$ be the unique member of $A^{\prime \prime}$ such that there is a 0-definable bijection between $I$ and $I^{\prime}$, and let $J(I)$ be the image of $J\left(I^{\prime}\right)$ under that bijection. Recall that each $X \in \mathcal{F}_{l}$ is the union of $k_{l} E_{l}$-classes. Let $X^{-}$be constructed from $X$ by replacing each $E_{l}$-class $D \subseteq X$ by the unique $E_{l+1}$-class whose coordinates in $H_{D}$ are just the elements of $\bigcup\left\{J(I): I \in \not_{D}^{\prime}\right\}$. Let $k_{l+1}=k_{l}$ and $\mathcal{F}_{l+1}=\left\{X^{-}: X \in \mathcal{F}_{l}\right\}$. We must verify that $\mathcal{F}_{l+1}$ is a replacement for $\mathcal{F}_{l}$ in $\mathcal{M}$. The main point to notice is that, if we choose $\mathcal{H}^{\prime \prime}$ and the sets $J(I)$ differently, then there exists $\alpha \in G(\mathscr{H})$ taking one possibility for $\mathcal{F}_{l+1}$ onto the other. This follows from the mutual quasi-indiscernibility of $\not^{\prime \prime}$. Note that each $\alpha \in G(\mathcal{H})$ fixes setwise each $D \in M / E_{l}$ which is included in a member of $\mathcal{F}_{l}$. Let $\alpha \in G$ induce a permutation $\alpha$ of $\mathcal{F}_{l}$. If $D \in M / E_{l}$ and $D \subseteq X \in \mathcal{F}_{l}$, then $\alpha(D) \in M / E_{l}, \alpha(D) \subseteq \alpha(X), \alpha\left(H_{D}\right)=\not_{\alpha(D)}$, and $\alpha(\not{H})=\not{H}$. It follows 
that $\alpha\left(\mathscr{H}^{\prime}\right)=\mathscr{H}^{\prime}$. Hence the family $\left\{\alpha(J(I)): I \in \mathscr{H}^{\prime}\right\}$ could have been obtained as $\left\{J(I): I \in \mathscr{H}^{\prime}\right\}$ if the appropriate choices had been made. Therefore we can find $\beta \in G(\not{H})$ such that $\beta \alpha(J(I))=J(\alpha(I))$ for all $I \in \not H$. It is clear that $\beta \alpha$ induces the same permutation of $\mathcal{F}_{l}$ as $\alpha$ and $\beta \alpha$ also induces a permutation of $\mathcal{F}_{l+1}$. This completes the proof that $\mathcal{F}_{l+1}$ is a replacement for $\mathcal{F}_{l}$ in $\mathcal{M}$.

We have shown how to obtain bounds for the $k_{l}$ which depend only on $i$. This gives us a bound on $e=k_{j}+1$ which depends only on $i$, and hence a bound on $F(e)=|\mathcal{F}|$. Thus in the last case, where $\mathcal{N}_{l}$ is quasi-coordinatizable and $\operatorname{dim}\left(\mathcal{N}_{l}\right) \geq$ $i$, we can compute $H(i)$ so that $\operatorname{dim}\left(\mathcal{N}_{l}\right)$ will be large enough provided $\operatorname{dim}\left(\mathcal{N}_{l}\right)>$ $H(i)$.

This completes the proof of Lemma A.

2.3. Theorem 2 implies Theorem 1 . Let $\mathcal{M}=(M ; G)$ be a permutation structure. For $C \subseteq M$ we define the rank of $C$ in $\mathcal{M}$, denoted $\operatorname{rk}_{\mathcal{M}}(C)$, as follows.

DEFINITION. If $C=\varnothing, \operatorname{rk}_{\mathcal{M}}(C)=-1$. If $C \neq \varnothing, \operatorname{rk}_{\mathcal{M}}(C)$ is the greatest $n \in \omega$ such that there exist sets $D_{\eta} \subseteq M\left(\eta \in{ }^{<m}\right)$ increasing in $\eta$ and elements $c_{\eta} \in C$ $\left(\eta \in{ }^{n} 2\right)$, all in the same orbit of $G$, such that for all $\eta, \xi \in{ }^{n} 2$ and $\sigma \subseteq \eta, \xi, c_{\eta}$ and $c_{\xi}$ are in the same orbit of $G\left(D_{\sigma}\right)$ iff $\sigma \neq \eta \cap \xi$. Here $\eta \cap \xi$ is the greatest common initial segment of $\eta, \xi$. For $n=0$ the condition is satisfied trivially. If there is no greatest $n$, we set rk $_{\mathcal{M}}(C)=\infty$.

This definition is required for technical reasons. Our main interest is in the rank of a structure. We define $\operatorname{rk}(\mathcal{M})=\operatorname{rk}_{\mathcal{M}}(M)$. This rank measures the complexity of the structure $\mathcal{M}$. If $\mathcal{M}$ is a countable $\aleph_{0}$-categorical structure, amongst which we count all finite structures, then $\operatorname{rk}(\mathcal{M})$ is the same as

$$
\sup \{\operatorname{CR}(p, 2): p \text { is a complete 1-type of } \operatorname{Th}(\mathcal{M})\},
$$

where $\mathrm{CR}(p, 2)$ is the complete rank of $[20$, p. 55].

For the rest of the section fix a finite relational language $L$. Let $a_{L} \in \omega$ be the maximum arity of any of the relation symbols of $L$; we call this the arity of $L$. Let $\mathcal{M} \in \operatorname{Hom}(L)$. As remarked above $\operatorname{Th}(\mathcal{M})$ admits elimination of quantifiers. Hence every $L$-formula is equivalent to a Boolean combination of atomic and negated atomic formulas. Moreover, since $L$ is finite the atomic and negated atomic formulas are all instances of a finite set of formulas. From these remarks it is easy to deduce that, if $\mathcal{M}$ is stable and countable, then $\operatorname{rk}(\mathcal{M})<\omega$. Our definition of rank is not appropriate for uncountable $\mathcal{M}$. If we use the model-theoretic definition of rank which agrees with our definition for countable structures, then

$$
\operatorname{rk}(\mathcal{M})=\max \{\operatorname{rk}(\mathcal{N}): \mathcal{N} \leq \mathcal{M}, \mathcal{N} \text { countable }\} \text {. }
$$

In the rest of the section we shall assume that all structures mentioned are countable; this is only for technical convenience. Recall that $\operatorname{Hom}(L, \omega)$ is the class of all stable $\mathcal{M} \in \operatorname{Hom}(L)$, while $\operatorname{Hom}(L, r)=\{\mathcal{M} \in \operatorname{Hom}(L): \operatorname{rk}(\mathcal{M}) \leq r\}(r<\omega)$.

Given Theorem 2, our purpose is to prove

THEOREM 1. There exists $r \in \omega$ such that $\operatorname{Hom}(L, \omega)=\operatorname{Hom}(L, r)$.

PROOF. We state two lemmas, prove the theorem from them, and then prove the lemmas.

Lemma 5. If $\mathcal{M} \in \operatorname{Hom}(L, \omega)$ and $A, B \subseteq M$, then

$$
\operatorname{rk}_{\mathcal{M}}(A \cup B) \leq \operatorname{rk}_{\mathcal{M}}(A)+\operatorname{rk}_{\mathcal{M}}(B)+1 \text {. }
$$


LEMMA 6. There exists $F: \omega \rightarrow \omega$ such that if $\mathcal{M}=(M ; G) \in \operatorname{Hom}(L ; \omega)$, $E_{0}>E_{1}$ are 0 -definable equivalence relations on some orbit $C$ of $G, C_{i} \in C / E_{i}$ $(i<2)$, and the structure $\mathcal{N}$ induced on $C_{0} / E_{1}$ by $G\left(\left\{C_{0}\right\}\right)$ is coordinatizable, then $\operatorname{rk}_{\mathcal{M}}\left(C_{0}\right)<F\left(\operatorname{rk}_{\mathcal{M}}\left(C_{1}\right)\right)$.

Let $\mathcal{M}=(M ; G) \in \operatorname{Hom}(L ; \omega)$. We have to show how to bound $\operatorname{rk}(\mathcal{M})$ in terms of $L$. Choose a maximal sequence $C_{0}, \ldots, C_{i}$ such that $C_{i}$ is an orbit of $G$ on $M$, $C_{j} \subsetneq C_{j+1}$ for all $j<i$, and $C_{j}$ is a class of some 0 -definable equivalence relation on $M$ for all $j<i$. The number of possibilities for $C_{i}$ is bounded in terms of $L$. Thus by Lemma 5 it suffices to show that $\operatorname{rk}_{\mathcal{M}}\left(C_{i}\right)$ is bounded in terms of $L$. Since $i<s_{2}(\mathcal{M}), i$ is bounded in terms of $L$. Therefore it is enough to bound the $\operatorname{rk}_{\mathcal{M}}\left(C_{j+1}\right)$ in terms of $\operatorname{rk}_{\mathcal{M}}\left(C_{j}\right)$ and $L$. For $j \leq i$ let $E_{j}$ denote the unique 0-definable equivalence relation on $C_{i}$ such that $C_{j} \in C_{i} / E_{j}$.

Fix $j<i$ and let $\mathcal{N}$ be the structure induced on $C_{j+1} / E_{j}$ by $G\left(\left\{C_{j+1}\right\}\right)$. If $\mathcal{N}$ is coordinatizable $\operatorname{rk}_{\mathcal{M}}\left(C_{j+1}\right)<F\left(\operatorname{rk}_{\mathcal{M}}\left(C_{j}\right)\right)$ by Lemma 6 . Otherwise, by Theorem 2 , which is applicable since we are working within a single orbit, $\left|C_{j+1} / E_{j}\right|$ is bounded in terms of $L$, i.e., $C_{j+1}$ is the union of a bounded number of copies of $C_{j}$. In this case $\operatorname{rk}_{\mathcal{M}}\left(C_{j+1}\right)$ is bounded in terms of $\operatorname{rk}_{\mathcal{M}}\left(C_{j}\right)$ and $L$ by Lemma 5 . This completes the proof of Theorem 1 .

We now turn to the proof of the lemmas. The first one is easy.

Proof of LemMa 5. Let $D_{\eta} \subseteq M\left(\eta \in{ }^{<n} 2\right)$ and $c_{\eta} \in A \cup B\left(\eta \in{ }^{n} 2\right)$ witness that $\operatorname{rk}_{\mathcal{M}}(A \cup B)=n$ according to the definition. Suppose that $\operatorname{rk}_{\mathcal{M}}(A)=m<n$. There exists $\sigma \in{ }^{m} 2$ and $\eta \in{ }^{n} 2$ such that $\sigma \subseteq \eta$ and the orbit of $G\left(D_{\sigma}\right)$ in which $c_{\eta}$ lies does not intersect $A$. Otherwise the given $D_{\eta}$ 's $\left(\eta \in{ }^{\prime m} 2\right)$ together with new $c_{\eta}$ 's $\left(\eta \in{ }^{m+1} 2\right)$ witness that $\operatorname{rk}_{\mathcal{M}}(A)>m$. Without loss suppose $\tau=\sigma^{-}(0) \subseteq \eta$. Define $D_{\eta}^{\prime}=D_{\sigma \frown(0)-\eta}\left(\eta \in<^{<n-m-1} 2\right)$ and $c_{\eta}^{\prime}=c_{\sigma \sim(0)-\eta}\left(\eta \in^{n-m-1} 2\right)$. All the $c_{\eta}^{\prime}$ are in $B$ and together with the $D_{\eta}^{\prime}$ they witness $\operatorname{rk}_{\mathcal{M}}(B) \geq n-m-1$. This completes the proof of Lemma 5.

Before proving Lemma 6 we state without proof another lemma which is an easy consequence of the definition of rank.

Lemma 7. Let $\mathcal{M}=(M ; G) \in \operatorname{Hom}(L, \omega)$ and $C \subseteq M$. If $\operatorname{rk}_{\mathcal{M}}(C) \geq j+j^{\prime}$, then there exist $D_{\eta} \subseteq M\left(\eta \in{ }^{<j} 2\right)$ and $P_{\eta} \subseteq M\left(\eta \in \overline{\leq j}_{2}\right)$ such that the following conditions are satified:

(i) $P_{()}$is an orbit of $G$ on $M$.

(ii) $\left|D_{()}\right|<a_{L}$.

(iii) $D_{\eta} \subseteq D_{\eta^{-(i)}}$ and $\left|D_{\eta_{-(i)}} \backslash D_{\eta}\right|<a_{L}\left(\eta \in^{j-1} 2, i<2\right)$.

(iv) $P_{\eta^{-(i)}} \subseteq P_{\eta}, P_{\eta^{-(i)}}$ is an orbit of $G\left(D_{\eta}\right)$ on $M$, and $P_{\eta^{-(0)}} \cap P_{\eta^{-(1)}}=\varnothing$ $\left(\eta \in{ }^{<j} 2, i<2\right)$.

(v) $\operatorname{rk}_{\mathcal{M}}\left(C \cap P_{\eta}\right) \geq j^{\prime}\left(\eta \in{ }^{j} 2\right)$.

Proof of LemMa 6. Let $\mathcal{M}, C, E_{i}, C_{i}(i<2)$, and $\mathcal{N}$ be as in the statement of the lemma. There is a twisted coordinate system $\left(H_{\mathcal{N}} ; G_{\mathcal{N}}\right)$ for $\mathcal{N}$. Moreover, $H_{\mathcal{N}}$ can be found as a subset of $M^{\text {eq }}$ definable from the single parameter $C_{0}$ such that the action of $G_{\mathcal{N}}$ is exactly that induced by $G\left(\left\{H_{\mathcal{N}}\right\}\right)$, and such that $H_{\mathcal{N}}$ is partitioned into components by the restriction of a 0 -definable equivalence relation. For $a \in C_{0}$ let $\operatorname{crd}(a)$ denote the subset of $H_{\mathcal{N}}$ corresponding to $a / E_{1} \in N$. Recall that $|\operatorname{crd}(a)|=k=\operatorname{wd}(\mathcal{N}) \cdot \operatorname{ind}(\mathcal{N})$ for all $a \in C_{0}$ and, from Lemma 1 , that $k$ is 
bounded in terms of $L$. For $X \subseteq H_{\mathcal{N}}$ let $C_{0}(X)$ denote $\left\{a \in C_{0}: X \subseteq \operatorname{crd}(a)\right\}$. By induction on $k-|X|$ we will show how to bound $\operatorname{rk}_{\mathcal{M}}\left(C_{0}(X)\right)$ in terms of $\operatorname{rk}_{\mathcal{M}}\left(C_{1}\right)$.

Let $X \subseteq H_{\mathcal{N}}$. If $|X| \geq k$, then either $C_{0}(X)=\varnothing$ or $C_{0}(X)$ is conjugate to $C_{1}$ under $G$. In either case $\operatorname{rk}_{\mathcal{M}}\left(C_{0}(X)\right) \leq \operatorname{rk}_{\mathcal{M}}\left(C_{1}\right)$. Let $Y \subseteq H_{\mathcal{N}},|Y|<k$, and for induction suppose that a bound $j$ for $\operatorname{rk}_{\mathcal{M}}\left(C_{0}(X)\right)$, good whenever $|Y|<|X|$, has already been found. It is sufficient to find a bound for $\operatorname{rk}_{\mathcal{M}}\left(C_{0}(Y)\right)$ in terms of $j$ and $\operatorname{rk}_{\mathcal{M}}\left(C_{1}\right)$. Let $\forall$ be the set of components of $H_{\mathcal{N}}$. Note that there is a natural bijection between $\forall$ and a subset of $M^{\text {eq }}$ defined from the single parameter $C_{0}$. From Lemma $1,|\mathcal{H}|=\operatorname{wd}(\mathcal{N})$ is bounded. Thus fixing $I \in \mathcal{H}$ it is sufficient to bound the rank of $\mathcal{M}$ of

$$
C_{0}(Y, I)=\left\{a \in C_{0}(Y):(\operatorname{crd}(a) \backslash Y) \cap I \neq \varnothing\right\}
$$

in terms of $j$ and $\operatorname{rk}_{\mathcal{M}}\left(C_{1}\right)$.

Let $D_{\eta} \subseteq M\left(\eta \in{ }^{<j} 2\right)$ and $P_{\eta} \subseteq M\left(\eta \in{ }^{\leq j} 2\right)$ satisfy the conclusion of Lemma 7 with $C_{0}(Y, I)$ instead of $C$ and $j^{\prime}=\operatorname{rk}_{\mathcal{M}}\left(C_{0}(Y, I)\right)-j$. Let $B=Y \cup \bigcup\left\{D_{\eta}: \eta \in^{<j} 2\right\}$. From Lemma 4 (extended to allow $|I|=\aleph_{0}$ if necessary) either $|I|$ is bounded in terms of $|B|$ and hence in terms of $j$, or $I-\operatorname{cl}_{\mathcal{M}}(B)$ exists and $I-\operatorname{cl}_{\mathcal{M}}(B)$ is bounded in terms of $j$. In the first case, since

$$
C_{0}(Y, I)=\bigcup\left\{C_{0}(Y \cup\{b\}): b \in I \backslash Y\right\}
$$

we get a bound on $\operatorname{rk}_{\mathcal{M}}\left(C_{0}(Y, I)\right)$ in terms of $j$ from Lemma 1 . In the second case for any $b \in I \backslash I-\operatorname{cl}(B)$ there exists $\eta \in{ }^{j} 2$ such that $P_{\eta} \cap C_{0}(Y \cup\{b\})=\varnothing$ since $\operatorname{rk}_{\mathcal{M}}\left(C_{0}(Y \cup\{b\})\right)<j$ by choice of $j$. Since $G(B)$ fixes $P_{\eta}$ setwise and acts transitively on $I \backslash I-\operatorname{cl}(B)$, one $\eta$ is good for all $b \in I \backslash I-\operatorname{cl}(B)$. Thus

$$
P_{\eta} \cap C_{0}(Y, I) \subseteq \bigcup\left\{C_{0}(Y \cup\{b\}): b \in I-\operatorname{cl}_{\mathcal{M}}(B) \backslash Y\right\} .
$$

By Lemma 5 we have a bound on $\operatorname{rk}_{\mathcal{M}}\left(P_{\eta} \cap C_{0}(y, I)\right)$ in terms of $j$ since $\mid I$-cl $\mathrm{M}_{\mathcal{M}}(B) \mid$ is already bounded in terms of $j$. Therefore we have bounded the values of $j^{\prime}$ which completes the proof.

3. Finite permutation structures. In this section we make the convention that all groups and structures we consider are finite. A fair amount of background material has been included for the benefit of model theorists unused to dealing with finite simple groups. Part of this material is included in the survey [5] which deals with the impact of the classification of the finite simple groups on problems very like the one with which we are presently dealing. An introduction to the study of permutation groups can be found in Chapter 1 of $[\mathbf{1 7}]$.

Some of our notations are as follows. Let $G$ be a group acting on a set $M$ (i.e., $G \leq \operatorname{Sym}(M)$ or we have in mind a particular homomorphism $\varphi: G \rightarrow \operatorname{Sym}(M))$. Let $x, g \in G$ and $X \subseteq G$. Then $x^{g}, X^{g}$ denotes $g x g^{-1}, g X g^{-1}$ respectively. If $a \in M$, then $a^{G}$ denotes the $G$-orbit of $a$, i.e., $\{g(a): g \in G\}$, and $G_{a}$ denotes the stabilizer of $a$, i.e., $\{g \in G: g(a)=a\}$. If $A \subseteq M$, then $G(\{A\})$ denotes $\{g \in$ $G: g(A)=A\}$, the setwise stabilizer of $A$ in $G$, and $G(A)$ denotes $\bigcap\left\{G_{a}: a \in A\right\}$, the pointwise stabilizer. For any group $H, Z(H)$ denotes the centre of $H$ and $H^{\prime}$ the derived subgroup.

3.1. The O'Nan-Scott lemma. A key result discussed in [5] is a lemma of O'Nan and Scott (independently) which describes the action of the socle of a primitive 
group. In the corrected form given in [2] it may be stated thus:

LEMMA 8. Let $\mathcal{M}=(M ; G)$ be a primitive permutation structure, $a \in M$, $N=\operatorname{dfn} \operatorname{Soc}(G)$ be nonabelian, and $N_{a}$ denote $N \cap G_{a}$. Then there are isomorphic nonabelian simple groups $S_{i} \leq N(i \in I)$ such that

$$
N=\prod_{i \in I} S_{i} \quad(\text { direct product })
$$

and $N_{a}$ has one of the three forms:

1. Trivial.

2. A product of diagonal subgroups $D_{k} \leq \prod_{i \in I(k)} S_{i}$, where $\{I(k): k \in K\}$ partitions $I$.

3. $\prod_{i \in I} S_{i a}$, where $(1)<S_{i a}=_{\mathrm{dfn}} S_{i} \cap G_{a}<S_{i}$.

REMARK. 1. When $\mathcal{M}$ is a primitive permutation structure falling under Cases 1, 2 above, $|M|$ is bounded in terms of $s_{2}(\mathcal{M})$. In Case 2 this is explained in [5]. In Case 1 we can identify $M$ with $N$ by $g(a) \mapsto g(g \in N)$, and write $G=N G_{a}$. $N$ must act transitively on $M$ since $\mathcal{M}$ is primitive. Under the identification of $M$ with $N, G_{a}$ acts by conjugation by the inverse. Thus $s_{1}(N) \leq s_{2}(\mathcal{M})$, where on the left-hand side $N$ is seen as the structure $(N ; \operatorname{Aut}(N))$. Let $S$ be one of the simple factors of $N$. Clearly $|I|<s_{1}(N)$ and $s_{1}(S) \leq s_{1}(N)$. From the classification of finite simple groups, for a finite nonabelian simple group $S,|S|$ is bounded in terms of $s_{1}(S)$. (Cameron [5] mentions this and uses it in treating Case 2. For $S$ a Chevalley group one can see that $|S|$ is bounded in terms of $s_{1}(S)$ by looking at the diagonal elements when $S$ is represented as a group of matrices relative to a Chevalley basis of the simple Lie algebra on which $S$ acts.)

In our context a finite relational language $L$ is fixed and $(M ; G)$ is the permutation structure corresponding to $\mathcal{M} \in \operatorname{Hom}(L)$. Since $s_{2}(\mathcal{M})$ is bounded by the choice of $L$, Cases 1 and 2 never require any analysis when Lemma 8 is applied below because we know in these cases that $|M|$ is small.

2. Following [5], when Case 3 of the lemma obtains we can say a lot about the permutation structure $\mathcal{M}$. Let $|I|=d$. There are a primitive permutation structure $(X ; K)$ with $T=\operatorname{Soc}(K) \simeq S$, and a group $H$ with

$$
T^{d} \unlhd H \leq K \mathrm{Wr} \operatorname{Sym}(d) \leq \operatorname{Sym}\left(X^{d}\right)
$$

such that $(M ; G) \cong\left(X^{d} ; H\right)$ and $K$ is the group induced by $H$ on each of the $d$ copies of $X$. Here $T^{d}$ acts coordinatewise and $K \mathrm{Wr} \operatorname{Sym}(d)$ has the usual product action on $X^{d}$.

For $i \in I,\left\{S_{i a}: a \in M\right\}$ may be take as the $i$ th copy of $X$. Then $(a, g) \mapsto$ $\left(\left\{S_{i a}: i \in I\right\}, h\right)$ is an isomorphism of $\mathcal{M}$ onto $\left(X^{d} ; H\right)$, where

$$
h\left(\left\{S_{i b}: i \in I\right\}\right)=\left\{\left(S_{i b}\right)^{g}: i \in I\right\}=\left\{S_{i g(b)}: i \in I\right\} \quad(b \in M) .
$$

Let $S$ denote one of the $S_{i}$ 's and $S_{a}=S \cap G_{a}$. Then $X=\left\{S_{a}: a \in M\right\} ; T=$ $\left\{t_{s}: s \in S\right\} \leq \operatorname{Sym}(X)$, where $t_{g}(Y)=Y^{g}(Y \in X)$; and $K=\left\{t_{g}: g \in G, S^{g}=S\right\}$. Now $s \mapsto t_{s}$ is an isomorphism of $S$ and $T$. It is clear that $T \unlhd K$ and hence that $T$ is a minimal normal subgroup of $K$. If $S^{g}=S$ and $t_{g}$ centralizes $T$, then $Y^{g^{-1} s^{-1} g s}=Y(Y \in X)$, i.e., $\left(S_{a}\right)^{g^{-1} s^{-1} g s}=S_{a}(a \in M)$ for all $s \in S$. It follows that $g^{-1} s^{-1} g s(a)=a(a \in M, s \in S)$. Hence $t_{g}=1_{X}$ and so $T=\operatorname{Soc}(K)$. 
Finally, if $S^{g}=S$ then $t_{g} \in K$ can be identified with $\alpha_{g} \in \operatorname{Aut}(T)$ defined by $\alpha_{g}\left(t_{s}\right)=t_{g_{s g^{-1}}}(s \in S)$. Thus

$$
T=\operatorname{Soc}(K) \unlhd K \leq \operatorname{Aut}(T) .
$$

3. From $(M ; G) \cong\left(X^{d} ; H\right)$ it is clear that $s_{2}(\mathcal{M})>d$, because if $a, b \in X^{d}$ agree on exactly $j$ coordinates, so do $h(a), h(b)$ for each $h \in H$.

From Lemma 8 and the Remarks we formulate the following consequence of the O'Nan-Scott lemma needed below.

LEMMA 9. There is a function $m: \omega \rightarrow \omega$ with the following property. Let $\mathcal{M}=(M ; G)$ be a primitive permutation structure, $N=\operatorname{Soc}(G)$ be nonabelian, and $|M|>m\left(s_{2}(\mathcal{M})\right)$. Let $b \in M$ and $S$ be a simple factor of $N$. There is a primitive homogeneous substructure $(X ; K)$ of $\mathcal{M}$ such that $X=b^{S}, S \cong T={ }_{\mathrm{dfn}} \operatorname{Soc}(K) \unlhd$ $K \leq \operatorname{Aut}(T)$, and $|X|^{s_{2}(\mathcal{M})}>|M|$.

Proof. We choose $m: \omega \rightarrow \omega$ such that, when Lemma 8 is applied to $\mathcal{M}$ satisfying $|M|>s_{2}(\mathcal{M})$, Case 3 obtains, i.e., $N_{a}=\prod_{i \in I} S_{i a}$ where (1) $<S_{i a}<S_{i}$. Let $S$ be $S_{j}$ and $S_{j a}$ be denoted $S_{a}$. Let

$$
X=\left\{a \in M: S_{i a}=S_{i b}(i \in I \backslash\{j\})\right\} .
$$

If the mapping $a \mapsto N_{a}(a \in M)$ is constant, $N_{a} \unlhd N$ and so (1) $<S_{i a} \triangleleft S_{i}$, a contradiction. Hence the mapping $a \mapsto N_{a}(a \in M)$ is one-to-one, because $\mathcal{M}$ is primitive. By the same token $N$ acts transitively on $M$. For each $a \in M$ there exists $g \in N$ such that $\left(N_{b}\right)^{g}=N_{a}$. Thus for each $a \in M$ there exists $s \in S$ such that $\left(S_{b}\right)^{s}=S_{a}$. It follows that $X=b^{S}$.

Let $a_{0}, a_{1} \in X, a_{0} \neq a_{1}, g \in G$, and $g\left(a_{0}\right), g\left(a_{1}\right) \in X$. Clearly $S_{a_{0}} \neq S_{a_{1}}$. If $S^{g}=S_{1}$ and $i \neq j$, then $S_{i g\left(a_{0}\right)}=\left(S_{a_{0}}\right)^{g} \neq\left(S_{a_{1}}\right)^{g}=S_{i g\left(a_{1}\right)}$ which contradicts $g\left(a_{0}\right), g\left(a_{1}\right) \in X$. Therefore $S^{g}=S$. It follows that

$$
\left\{\left(S_{i b}\right)^{g}: i \in I \backslash\{j\}\right\}=\left\{S_{i b}: i \in I \backslash\{j\}\right\} .
$$

Hence $g(X)=X$. Since we have already seen that $S$ acts transitively on $X, X$ is the universe of a homogeneous substructure $(X ; K)$ of $\mathcal{M}$. If we identify $a \in X$ with $S_{a}$, thus identifying $X$ with its namesake from Remark 2 , then $K$ will be the same subgroup of $\operatorname{Sym}(X)$ as before. Thus

$$
S \cong T=_{\mathrm{dfn}} \operatorname{Soc}(K) \unlhd K \leq \operatorname{Aut}(T) .
$$

Since $|X|^{d}=|M|$ and $d<s_{2}(\mathcal{M}),|M|<|X|^{s_{2}(\mathcal{M})}$.

Above we considered primitive permutation structures $\mathcal{M}=(M ; G)$ such that $\operatorname{Soc}(G)$ is nonabelian. When $\operatorname{Soc}(G)$ is abelian the situation is deceptively simple:

LEMMA 10 . Let $\mathcal{M}=(M ; G)$ be a primitive permutation structure and $A=$ $\operatorname{Soc}(G)$ be abelian.

(i) $A$ acts regularly on $M$.

(ii) $A$ is an elementary abelian p-group for some prime $p$.

(iii) Fix $b \in M$ and let $H=G_{b} . M$ can be identified with $A$ by $a(b) \mapsto a(a \in A)$ and under this identification $A$ acts by translation and $H$ by conjugation by the inverse. Further $G=A H$ and $A \cap H=(1)$.

The proof is easy; this is one of the basic lemmas of the theory of permutation groups. 
3.2. The classical simple groups. Via the O'Nan-Scott lemma we become concerned with simple groups. In this section we establish some notation and review briefly some basic information required in the sequel.

Let $V$ be a vector space over a finite field $F_{q}$. With $V$ are associated certain groups of linear transformations $\mathrm{SL}(V), \mathrm{Sp}(V), \mathrm{SU}(V), \Omega^{ \pm}(V)$. In the case of $\mathrm{Sp}(V), \mathrm{SU}(V), \Omega^{ \pm}(V)$ the vector space $V$ is equipped with a scalar product $[u, v]$ which is nonsingular, (i.e., $V^{\perp}=(0)$ ) except in the case of $\Omega^{ \pm}(V)$ with $q$ even and $\operatorname{dim}(V)$ odd. When there is a scalar product present, for each subspace $U \leq V$, $U^{\perp}$ denotes $\{v \in V:[v, u]=0$ for all $u \in U\}$. Except in the case of $\Omega^{ \pm}(V)$ just mentioned, $\operatorname{dim}(U)+\operatorname{dim}\left(U^{\perp}\right)=\operatorname{dim}(V)$. In that special case $V^{\perp}$, called the radical, has dimension 1 . Hence $\operatorname{dim}(U)+\operatorname{dim}\left(U^{\perp}\right)$ is either $\operatorname{dim}(V)$ or $\operatorname{dim}(V)+1$.

A subspace $U \leq V$ is nonsingular if the restriction of the scalar product to $U$ is nonsingular, and totally isotropic if $U \leq U^{\perp} . U$ is nonsingular modulo $V^{\perp}$ if $V^{\perp} \leq U$ and $U^{\perp}=V^{\perp}$.

In the case of $\Omega^{ \pm}(V)$ with $q$ even the vector space is equipped not only with a scalar product but also with a quadratic form $Q(v)$, i.e., a mapping from $V$ into $F_{q}$ satisfying

$$
Q(\lambda u+\mu v)=\lambda^{2} Q(u)+\mu^{2} Q(v)+\lambda \mu[u, v]
$$

for all $\lambda, \mu \in F_{q}$ and $u, v \in V$. If $v \in V^{\perp} \backslash\{0\}$, then $Q(v) \neq 0$. We call $v \in V$ singular if $Q(v)=0$. A subspace $U \leq V$ is called totally singular if $Q(v)=0$ for all $v \in U$; such a subspace is clearly totally isotropic.

If $\operatorname{dim}(V)$ is even there are two different groups $\Omega^{+}(V)$ and $\Omega^{-}(V)$ arising from different scalar products (quadratic forms if $q$ is even). If $\operatorname{dim}(V)$ is odd, there is only one so we may denote it by $\Omega(V)$.

Let $U, V$ be vector spaces over $F_{q}$ equipped with a scalar product (and possibly a quadratic form). A linear transformation $\tau: U \rightarrow V$ is an isometry if $\left[\tau\left(u_{0}\right), \tau\left(u_{1}\right)\right]=$ $\left[u_{0}, u_{1}\right]$ for all $u_{0}, u_{1} \in U$ (and $Q(\tau u)=Q(u)$ for all $\left.u \in U\right)$, and $\tau$ is nonsingular and onto. In the case of $\mathrm{SL}(V)$ an isometry is just a nonsingular linear transformation.

Let $G(V)$ denote any one of the groups $\mathrm{SL}(V), \mathrm{Sp}(V), \mathrm{SU}(V), \Omega^{ \pm}(V)$ and let $P G(V)$ denote $G(V) / Z(G(V))$. The classical simple groups are those of the form $P G(V)$. The group $G(V)$ is the derived subgroup of the group of isometries arising from the appropriate scalar product (and quadratic form).

When $q$ is even and $\operatorname{dim}(V)$ is odd, $2 m+1$ say, $\Omega(V)$ is the same as $O(V)$, the group of isometries, and isomorphic to $\operatorname{Sp}(U)$, where $\operatorname{dim}(U)=2 m$. Thus no simple groups are lost if we exclude the symplectic groups when $q$ is even, because $\operatorname{Sp}(U)$ is defined only when $\operatorname{dim}(U)$ is even. This point must be borne in mind in applying Lemma 12 below.

Each of the groups $G(V)$ has a companion $\Gamma(V)(\geq G(V))$ consisting of all nonsingular semilinear transformations preserving the scalar product and the quadratic form when they are present. When $G(V)$ is $\mathrm{SL}(V), \mathrm{Sp}(V), \mathrm{SU}(V), \Omega^{ \pm}(V)$, then $\Gamma(V)$ is $\Gamma \mathrm{L}(V), \Gamma \mathrm{Sp}(V), \Gamma \mathrm{U}(V), \Gamma \mathrm{O}^{ \pm}(V)$ respectively. $P \Gamma(V)$ denotes $\Gamma(V) / Z \Gamma(V)$, the corresponding projective group.

We think of $G(V), \Gamma(V)$ as permutation groups acting on $V$, and of $P G(V)$, $P \Gamma(V)$ as permutation groups acting on the set of all subspaces of $V$.

When $G(V)$ is $\mathrm{SL}(V)$ for any subspace $U \leq V$ let $U^{\perp}$ denote the "orthogonal complement" of $U$ with respect to the usual inner product. Let $\mathrm{P} L^{+}(V)$ denote the group of permutations of the subspaces of $V$ generated by $\operatorname{P\Gamma L}(V)$ and $\sigma$ where 
$\sigma(U)=U^{\perp}$. Now $\operatorname{Aut}(\operatorname{PSL}(V))=\mathrm{P} L^{+}(V)$, i.e., each automorphism of $\operatorname{PSL}(V)$ is obtained by conjugating with respect to a suitable element of $\mathrm{P}^{+} \mathrm{L}^{+}(V)$ and the action of $\mathrm{P}^{+}(V)$ is faithful. Note that $\left|\mathrm{P}^{+} L^{+}(V): \mathrm{P} \Gamma \mathrm{L}(V)\right|=2$ if $\operatorname{dim}(V)>2$. In the other cases $\operatorname{Aut}(P G(V))=P \Gamma(V)$.

We now list the facts about the geometry of classical groups which will be needed below. These can be culled from [1, Chapter 3 and $\mathbf{9}$, Chapter 1]. Let $V$ be the vector space associated with $G(V)$, one of the groups listed above (other than $\mathrm{SL}(V))$.

Fact 1. Let $U \leq V$ be nonsingular, or totally isotropic (totally singular if a quadratic form is present). There are linearly independent $e_{1}, f_{1}, e_{2}, f_{2}, \ldots, e_{k}, f_{k} \in$ $V$ spanning $W \leq V$ with the following properties:

(a) $\left[e_{i}, f_{i}\right]=1,\left[f_{i}, e_{i}\right]=1$ (except in the symplectic case when the value is -1 ) $(1 \leq i \leq k)$; all the other scalar products between these vectors are 0 ; and $e_{i}, f_{i}$ are singular if there is a quadratic form.

(b) $\operatorname{dim}(V) \leq 2 k+4$, and for some $l \leq k, U \cap W$ is either $\left\langle e_{1}, f_{1}, \ldots, e_{l}, f_{l}\right\rangle$ or $\left\langle e_{1}, \ldots, e_{l}\right\rangle$.

(c) The isometries of $V$ determined by fixing $W^{\perp}$ pointwise, and either taking an even permutation of the pairs $\left(e_{1}, f_{1}\right), \ldots,\left(e_{k}, f_{k}\right)$, or mapping $e_{i}, f_{i}$ to $\left[e_{i}, f_{i}\right] f_{i}$, $\left[f_{i}, e_{i}\right] e_{i}$ for an even number of $i$ and fixing all the other basis vectors, belong to $G(V)$.

Fact 2. If $U \leq V$ is totally isotropic and $U \cap V^{\perp}=(0)$, there exists nonsingular $W$ such that $U \leq W \leq V$ and $\operatorname{dim}(W)=2 \operatorname{dim}(U)$.

Fact 3. Given a nonsingular subspace $U$ there exist a direct orthogonal decomposition $V=U_{1} \perp \cdots \perp U_{k} \perp W$ and isometries $\tau_{i}: U \rightarrow U_{i}$ such that $\operatorname{dim}(W)<\operatorname{dim}(U)+2$, and for any even $\pi \in \operatorname{Sym}(\{1, \ldots, n\})$ the isometry of $V$ determined by $\sigma \nmid W=\operatorname{id}_{W}, \sigma \uparrow U_{i}=\tau_{\pi(i)} \tau_{i}^{-1}(1 \leq i \leq k)$ belongs to $G(V)$.

Fact 4 (Witt's Theorem). If $U_{0}, U_{1} \leq V, \tau: U_{0} \rightarrow U_{1}$ is an isometry, and $\operatorname{dim}\left(U_{0}\right)=\operatorname{dim}\left(U_{1}\right)<(\operatorname{dim}(V)-3) / 2$, then there is an element of $G(V)$ extending $\tau$.

Fact 5. Let $q$ be even, $\operatorname{dim}(V)$ be odd, and $G(V)$ be $\Omega(V)$ which is the same as $O(V)$ in this case. If $U \leq V$ is nonsingular and $\operatorname{dim}(U)=\operatorname{dim}(V)-1$, we can choose a basis $e_{1}, \ldots, e_{l}, f_{1}, \ldots, f_{l}, d$ of $V$ such that

$$
Q\left(\Sigma\left(\lambda_{i} e_{i}+\mu_{i} f_{i}\right)+\nu d\right)=\Sigma \lambda_{i} \mu_{i}+\nu^{2}
$$

the scalar product is given by $[u, v]=Q(u+v)+Q(u)+Q(v), V^{\perp}=\langle d\rangle$, and $U$ is one of $\left\langle e_{1}, f_{1}, \ldots, e_{l}, f_{l}\right\rangle,\left\langle e_{1}, f_{1}, \ldots, e_{l-1}, f_{l-1}, e_{l}+d, f_{l}+d\right\rangle$.

3.3. The Chevalley groups. The classical simple groups are included in a larger class of simple groups comprising the Chevalley groups. These are the simple groups of Lie type studied in Carter [6]. Each of these groups is over a field $F_{q}$ which in the case of a classical group is the same field as before. Each of these groups also has a "rank". In the case of a classical group the dimension of the associated vector space $V$ is a strictly monotonic function of the rank, where the function depends on the kind of group. The nonclassical Lie groups all have bounded rank.

The classification of simple groups tells us that all but a finite number fall into one of three classes: the cyclic groups of prime order, the alternating groups, and the groups of Lie type. 
3.4. Primitive permutation structures with nonabelian simple socle. Much is known about primitive permutation structures $\mathcal{M}=(M ; G)$ when $\operatorname{Soc}(G)$ is nonabelian and $s_{4}(\mathcal{M})$ is small compared with $|M|$. Here we collect some of this information which we shall need below.

The first lemma shows that in our context we need only be concerned with primitive structures $\mathcal{M}$ such that the simple factors of $\operatorname{Soc}(G)$ are either alternating groups or classical simple groups with large rank.

LEMMA 11. There is a function $q: \omega^{2} \rightarrow \omega$ with the following property. Let $\mathcal{M}=(M ; G)$ be a primitive structure, $\operatorname{Soc}(G)$ be simple, and $\operatorname{Soc}(G)$ be a possibly twisted Chevalley group over $F_{q}$ of rank $n$. Then $q \leq q\left(s_{4}(\mathcal{M}), n\right)$.

REMARK 1. Later in the statement of Lemma 13 we shall see that, if $|M|$ is sufficiently large compared with $s_{4}(\mathcal{M})$, then $q$ is bounded just in terms of $s_{4}(\mathcal{M})$.

REMARK 2. By making $|M|$ large enough compared with $s_{4}(\mathcal{M})$, we force $\operatorname{Soc}(G)$ to be large compared with $s_{4}(\mathcal{M})$, and hence $n$ to be large. Thus, when $|M|$ is sufficiently large compared with $s_{4}(\mathcal{M})$, $\operatorname{Soc}(G)$ must be a classical group.

ProOF. We will give the proof mainly for $\operatorname{Soc}(G)$ untwisted, with a small comment on the twisted case. The proof relies heavily on the theory of Chevalley groups described in Carter [6] from where we take all our notation. Essentially the same proof works for the twisted case, mutatis mutandis, but there are a lot of details to be checked which we omit.

There is a natural homomorphism of $G$ into $\operatorname{Aut}(\operatorname{Soc}(G))$ with kernel $C_{G}(\operatorname{Soc}(G))$ $=1$. Thus with some abuse of notation we can write $G \leq \operatorname{Aut}(\operatorname{Soc}(G))$. There is a function $k(n)$ such that the index of $\operatorname{Soc}(G)$ in $\operatorname{Aut}(\operatorname{Soc}(G))$ is $\leq k(n) \log _{2} q$. Below we will show that, after $G$ has been replaced by $\operatorname{Soc}(G)$, then $q / \log _{2} q \leq s_{4}(\mathcal{M})$. Thus for the original $\mathcal{M}$, we have $q / k(n)\left\{\log _{2} q\right\}^{2} \leq s_{4}(\mathcal{M})$ which is the desired conclusion. On this basis we shall suppose that $G=\operatorname{Soc}(G)$, i.e., that $G$ itself is simple.

When we replace $G$ by $\operatorname{Soc}(G), \mathcal{M}$ may cease to be primitive. However, $\mathcal{M}$ is still transitive since $\operatorname{Soc}(G) \unlhd G$. Let $E$ be a maximal $\operatorname{Soc}(G)$-invariant equivalence relation on $M$. Then $\operatorname{Soc}(G)$ acts primitively on $M / E$. Thus, replacing $\mathcal{M}$ by $\left(M / E ; G^{*}\right)$ where $G^{*} \leq \operatorname{Sym}(M / E)$ is the group induced by $\operatorname{Soc}(G)$, we recover the primitivity. Exchanging $M$ for $M / E$ does not increase $s_{4}(\mathcal{M})$.

The proof works as follows. We will find $M_{1} \subseteq M$ and $G_{1}^{*} \leq \operatorname{Sym}\left(M_{1}\right)$ such that

$$
\forall g \in G\left[\left|M_{1} \cap g\left(M_{1}\right)\right|>1 \rightarrow g\left(M_{1}\right)=M_{1}\right]
$$

$G_{1}^{*}$ is the group induced by $G$, and $\mathcal{M}_{1}=\left(M_{1} ; G_{1}^{*}\right)$ is the projective line over $F_{q}$ possibly augmented by some field isomorphisms. Clearly, $s_{4}\left(\mathcal{M}_{1}\right) \leq s_{4}(\mathcal{M})$ and $q / \log _{2}(q)<s_{4}\left(\mathcal{M}_{1}\right)$ since the projective line has more than $q 4$-types and $\log _{2}(q)$ is a bound for the index of $\operatorname{SL}(2, q)$ in its automorphism group. (When $G$ is twisted, instead of the projective line we will have a structure arising from the parabolic action of one of the rank 1 twisted groups ${ }^{2} A_{2}(q),{ }^{2} B_{2}(q),{ }^{2} G_{2}(q)$. By "parabolic action" here we mean that the structure is transitive and the stabilizer of a point is a conjugate of the Borel subgroup.)

To find $\mathcal{M}_{1}$ we shall use freely the notation and theory developed in [6]. Thus we have a root system $\Phi$ containing a fundamental system $\Pi$, a Borel subgroup $B$, unipotent subgroup $U$, diagonal subgroup $H$, root groups $X_{r}(r \in \Phi)$, etc. 
The mainspring of our proof is the theorem of Seitz $[18$, p. 508] which gives either the desired bound at once or $a \in M$ and parabolic $P$ such that $G_{a} \leq P<G$. Since $\mathcal{M}$ is primitive $G_{a}=P$ is a maximal parabolic subgroup. From [6, Theorem 8.3.4] there exists $r \in \Pi$ such that $P=B N_{I} B$, where $I=\Pi \backslash\{r\}$.

We now establish some notation. Let $M_{1}=a^{B\left\langle n_{r}\right\rangle B}, J=\{s \in \Pi: s \perp r\} \cup\{r\}$, $Q=B N_{J} B$, and $U_{0}=\left\langle X_{s}: s \in \Phi^{+} \backslash\{r\}\right\rangle$. Let $G_{1}=\left\langle X_{r}, X_{-r}\right\rangle$.

We prove four propositions:

(i) $M_{1}=\{a\} \cup\left\{x n_{r}(a): x \in X_{r}\right\}$.

(ii) If $g \in G$ and $\left|M_{1} \cap g\left(M_{1}\right)\right|>1$, then $g \in Q$.

(iii) $U_{0} \leq G\left(M_{1}\right)$ and $n_{s} \in G\left(M_{1}\right)(s \in J \backslash\{r\})$.

(iv) For each $g \in Q$ there exist $h \in H$ and $g_{1} \in G_{1}$ such that $h g_{1}(b)=g(b)$ $\left(b \in M_{1}\right)$.

Proof OF (i). From [6, 8.1.4], $B\left\langle n_{r}\right\rangle B=B n_{r} B \cup B$. Since $B \leq P, a^{B}=\{a\}$. Since $B=U H, H$ normalizes $U$, and $n_{r}$ normalizes $H$ (by $[6,7.2 .2]$ ), $B n_{r} B=$ $U H n_{r} B=U n_{r} H B=U n_{r} B$.

From the commutator formula $[6,5.2 .2], U=X_{r} U_{0}$ where $U_{0}=\left\langle X_{s}: s \in\right.$ $\left.\Phi^{+} \backslash\{r\}\right\rangle$. From [6, 7.2.1] and $[6,2.1 .5], n_{r} U_{0}=U_{0} n_{r}$. Therefore $U n_{r} B=$ $X_{r} U_{0} n_{r} B=X_{r} n_{r} U_{0} B=X_{r} n_{r} B$. The conclusion is now clear since $B \leq P=G_{a}$.

ProOF OF (ii). Let $g \in G$ and $\left|M_{1} \cap g\left(M_{1}\right)\right|>1$. We will show that $g \in Q$. Since $B\left\langle n_{r}\right\rangle B \leq Q$ acts transitively on $M_{1}$, without loss of generality $g(a)=a$, i.e., $g \in P$. Since $B \leq G_{a} \cap G\left(\left\{M_{1}\right\}\right)$ and $P=B N_{I} B$, without loss of generality $g \in N_{I}$. Indeed we may suppose $g=\Pi_{i<k} n_{i}$, where $n_{i} \in\left\{n_{s}: s \in I\right\}(i<k)$. Let $w$ be the image of $g$ under the natural homomorphism of $N$ onto $W$. Let roots in $\Phi$ be seen as linear combinations of the roots in $\Pi$. For any $t \in \Phi$ and $s \in \Pi \backslash\{r\}, w_{s}(t)$ and $t$ have the same coefficient of $r$. Hence $w(r) \in \Phi^{+}$. By hypothesis $g x n_{r}(a)=y n_{r}(a)$ for some $x, y \in X_{r}$. From [6, 7.2.1], $g x=z g$ for some $z \in X_{w(r)} \leq U$. Hence $B g x=B g$. Now we have

$$
B g n_{r} B \subseteq B g x n_{r} P=B y n_{r} P=B n_{r} B B N_{I} B \subseteq B n_{r} N_{I} B \cup B N_{I} B
$$

by $[6,8.1 .6]$. Also $B g n_{r} B \cap B N_{I} B \neq \varnothing$ implies $n_{r} \in P$, a contradiction since $G=B N G=\left\langle n_{r}, P\right\rangle$. Thus $B g n_{r} B \subseteq B n_{r} N_{I} B$. From [6, 8.2.3] there exists $n \in N_{I}$ such that $g n_{r}$ and $n_{r} n$ map into the same element of $W$. It follows that $\left(w_{r}\right)^{-1} w w_{r}=w_{r} w w_{r} \in W_{I}$, whence $w_{r} w w_{r}(r) \in \Phi^{+}$. As noted above $w(r) \in \Phi^{+}$. Hence $w w_{r}(r)=w(-r)=-w(r) \in \Phi^{-}$. Since $w_{r}(w(-r)) \in \Phi^{+}, w(-r)=-r$ by $[6$, 2.1.5] which means that $w(r)=r$. From $[6,2.5 .4] w$ is a product of reflections $w_{s}$ $\left(s \in \Phi_{I}, s \perp r\right)$. Each such $s$ may be written $\Sigma\left\{\lambda_{t} t: t \in \Pi \backslash\{r\}\right\}$, where the nonzero $\lambda_{t}$ are all positive or all negative. Hence

$$
(r, s)=\Sigma\left\{\lambda_{t}(r, t): t \in \Pi \backslash\{r\}\right\}=0 .
$$

But by [6, 2.1.4], $(r, t) \leq 0$ for all $t \in \Pi \backslash\{r\}$. Hence $\lambda_{t} \neq 0$ only if $(r, t)=0$, which means that $t \in J \backslash\{r\}$. Therefore $w_{s} \in W_{J \backslash\{r\}}\left(s \in \Phi_{I}, s \perp r\right)$, and so $w \in W_{J \backslash\{r\}}$. It follows that $g \in N_{J}$ as required.

PROOF OF (iii). Recall that $M_{1}=\{a\} \cup\left\{x n_{r}(a): x \in X_{r}\right\}$. Since $U_{0} \leq P$ and $n_{s} \in P(s \in J \backslash\{r\}), U_{0} \leq G_{a}$ and $n_{s} \in G_{a}(s \in J \backslash\{r\})$. Since $s \perp r(s \in J \backslash\{r\})$, $w_{s}(r)=r$ and $\eta_{s, r}=1$, whence $n_{s} x=x n_{s}\left(x \in X_{r}, s \in J \backslash\{r\}\right)$ by [6, 7.2.1]. By the same token, if $s \in J \backslash\{r\}$, then $\left[w_{r}, w_{s}\right]=1$ and so $n_{s} n_{r}=n_{r} n_{s} h$ for some $h \in H$ depending on $r, s$. Since $h, n_{s} \in P=G_{a}, n_{s} x n_{r}(a)=x n_{r}(a)\left(x \in X_{r}, x \in J \backslash\{r\}\right)$, 
i.e., $n_{s} \in G\left(M_{1}\right)(s \in J \backslash\{r\})$. Now consider $U_{0}$. From the commutator formula [6, 5.2.2] $U_{0} x=x U_{0}\left(x \in X_{r}\right)$. Also, as noted above, $U_{0} n_{r}=n_{r} U_{0}$. It follows that $U_{0} \leq G\left(M_{1}\right)$ which completes the proof.

PROOF OF (iv). Since $H \triangleleft N$ and $N_{J}$ is the group generated by $H$ and the $n_{s}$ $(s \in J)$, we have $N_{J}=H\left\langle n_{s}: s \in J\right\rangle$. In $W_{J},\left(w_{r}\right)^{2}=1$ and $\left[w_{r}, w_{s}\right]=1$ for all $s \in J \backslash\{r\}$. Since $H$ is the kernel of the natural map from $N_{J}$ onto $W_{J}$,

$$
N_{J}=H\left(n_{r}\left\langle n_{s}: s \in J \backslash\{r\}\right\rangle \cup\left\langle n_{s}: s \in J \backslash\{r\}\right\rangle\right) .
$$

Now

$$
\begin{aligned}
Q & =B N_{J} B=U H N_{J} H U=U H N_{J} U \\
& =U H\left(n_{r}\left\langle n_{s}: s \in J \backslash\{r\}\right\rangle \cup\left\langle n_{s}: s \in J \backslash\{r\}\right\rangle\right) U \\
& =H X_{r} U_{0}\left(n_{r}\left\langle n_{s}: s \in J \backslash\{r\}\right\rangle \cup\left\langle n_{s}: s \in J \backslash\{r\}\right\rangle\right) X_{r} U_{0} .
\end{aligned}
$$

For $s \in J \backslash\{r\}, n_{s} X_{r}=X_{r} n_{s}$ by $[6,2.1 .5]$ and $U_{0} X_{r}=X_{r} U_{0}$ since $U=X_{r} U_{0}$. Therefore

$$
Q=H\left(X_{r} n_{r} X_{r} \cup X_{r}\right) U_{0}\left\langle n_{s}: s \in J \backslash\{r\}\right\rangle U_{0} .
$$

Since $X_{r} n_{r} X_{r} \cup X_{r} \leq G_{1}$, we are done by (iii).

We are now ready to complete the argument. Let $\mathcal{M}_{1}=\left(M_{1} ; G_{1}^{*}\right)$, where $G_{1}^{*} \leq$ $\operatorname{Sym}\left(M_{1}\right)$ is the group induced by $G$. Since $M_{1}=a^{B\left\langle n_{r}\right\rangle B}$ and $H G_{1} \leq B\left\langle n_{r}\right\rangle B$, it follows from (iv) that $Q \leq G\left(\left\{M_{1}\right\}\right)$. From (ii) we have

$$
\forall g \in G\left[\left|M_{1} \cap g\left(M_{1}\right)\right|>1 \rightarrow g\left(M_{1}\right)=M_{1}\right]
$$

which gives $s_{4}\left(\mathcal{M}_{1}\right) \leq s_{4}(\mathcal{M})$.

From $[6,6.3 .1]$ there is a homomorphism of $\operatorname{SL}(2, q)$ onto $G_{1}$. Now $Z(\operatorname{SL}(2, q))$ has order 2 , and its sole nonidentity element $\left(\begin{array}{cc}-1 & 0 \\ 0 & -1\end{array}\right)$ maps onto $h_{r}(-1)$ in $G_{1}$. Sincee $h_{r}(-1) \in H \leq G_{a}, Z(\operatorname{SL}(2, q))$ acts trivially on $M_{1}=a^{G_{1}}$. Let $\varphi: H G_{1} \rightarrow$ $G_{1}^{*}$ be the natural homomorphism. Then $\varphi\left(G_{1}\right)$ is a copy of $\operatorname{PSL}(2, q)$ which acts transitively on $M_{1}$. Under the homomorphism of $\operatorname{SL}(2, q)$ onto $G_{1}, X_{r}$ is the image of the subgroup $\left\{\left(\begin{array}{ll}1 & t \\ 0 & 1\end{array}\right): t \in F_{q}\right\}$, and the image of the diagonal subgroup is $\leq$ $H$. Since $H, X_{r} \leq G_{a},\left(\varphi\left(G_{1}\right)\right)_{a}$ contains the Borel subgroup of $\varphi\left(G_{1}\right)$. The Borel subgroup is maximal. Hence $\left(\varphi\left(G_{1}\right)\right)_{a}$ is the Borel subgroup. Therefore $\left(M_{1} ; \varphi\left(G_{1}\right)\right)$ is a copy of the projective line. From $\left[6\right.$, p. 100] $H$ normalizes $G_{1}$. Hence $\varphi\left(G_{1}\right) \unlhd G_{1}^{*}$. We can identify elements of $M_{1}$ with the conjugates of the Borel subgroup of $\varphi\left(G_{1}\right)$ by $b \mapsto\left(\varphi\left(G_{1}\right)\right)_{b}$. Thus there is an embedding of $G_{1}^{*}$ in $\operatorname{Aut}\left(\varphi\left(G_{1}\right)\right)$ which extends the natural embedding of $\varphi\left(G_{1}\right)$ in $\operatorname{Aut}\left(\varphi\left(G_{1}\right)\right)$. This completes the proof of the lemma.

We wish to describe the possibilities for $\mathcal{M}=(M ; G)$ when $\operatorname{Soc}(G)$ is a classical simple group, $\mathcal{M}$ is a primitive permutation structure, and $|M|$ is large compared with $s_{4}(\mathcal{M})$. The key result is

LEMMA 12 (KANTOR [11, THEOREM 3]). There exists a function $n: \omega \rightarrow \omega$ such that the following is true. Let $\mathcal{M}=(M ; G)$ be a primitive permutation structure and $a \in M$. Let $V$ be a finite dimensional vector space over $F_{q}, \operatorname{dim}(V)>n\left(s_{2}(\mathcal{M})\right)$, and $(G(V), \Gamma(V))$ be one of the pairs $(\mathrm{SL}(V), \Gamma \mathrm{L}(V)),(\mathrm{Sp}(V), \Gamma \mathrm{Sp}(V))$ with $q$ odd, $(\mathrm{SU}(V), \Gamma \mathrm{U}(V)),\left(\Omega^{ \pm}(V), \Gamma \mathrm{O}^{ \pm}(V)\right)$. Further, let $\varphi: G \rightarrow P \Gamma(V)$ be an embedding such that $P G(V) \leq \varphi(G)$ and $P G(V) \$ \varphi\left(G_{a}\right)$. Then $\varphi\left(G_{a}\right)$ is the stabilizer in $\varphi(G)$ of a proper subspace of $V$. 
This lemma of Kantor easily leads us to

LEMMA 13. There exist $m: \omega \rightarrow \omega$ and $q: \omega \rightarrow \omega$ with the following property. Let $\mathcal{M}=(M ; G)$ be a primitive permutation structure such that $|M|>m\left(s_{4}(\mathcal{M})\right)$. Let $V$ be a finite dimensional vector space over $F_{q}, G(V)$ be one of the classical groups mentioned in Lemma 12, and $\varphi: \operatorname{Soc}(G) \rightarrow P G(V)$ be an isomorphism. Then there is an isomorphism $\psi: \mathcal{M} \rightarrow \mathcal{M}^{*}$, where $\mathcal{M}^{*}$ has one of the forms:

ST. 1. $\left(U^{P G(V)} ; G^{*}\right)$ where $U \leq V$ and $P G(V) \leq G^{*} \leq P \Gamma(V)$.

ST. 2. $\left(\{U, W\}^{\mathrm{PSL}(V)} ; G^{*}\right)$ where $G(V)=\mathrm{SL}(V), U, W \leq V, \operatorname{dim}(U)+$ $\operatorname{dim}(W)=\operatorname{dim}(V)$, either $U \cap W=(0)$ or $U \subseteq W$, and $\operatorname{PSL}(V) \leq G^{*} \leq \mathrm{P}^{+}(V)$.

Further, $U$ may be chosen such that one of the following two possibilities holds:

(a) $U$ is totally isotropic (totally singular if there is a quadratic form), or $G(V)=$ $\mathrm{SL}(V)$, or $U \geq V^{\perp}$ is nonsingular modulo $V^{\perp}, \operatorname{dim}(U) \leq 2 s_{2}(\mathcal{M})+3$.

(b) $G(V)=\Omega(V), q$ is even, $\operatorname{dim}(V)$ is odd, $U$ is nonsingular, $\operatorname{dim}\left(V^{\perp}\right)=1$, and $\operatorname{dim}(U)=\operatorname{dim}(V)-1$.

Finally, $q \leq q\left(s_{4}(\mathcal{M})\right)$.

Proof. Let $S=\operatorname{Soc}(G)$. From Remark 2 of 3.1, $S \unlhd G \leq \operatorname{Aut}(S)$ since $d=1$. We shall suppose that $|M|$ is large compared with $s_{4}(\mathcal{M})$ and draw the appropriate conclusions.

We first consider the case in which $\varphi$ can be extended to an embedding $\varphi: G \rightarrow$ $P \Gamma(V)$. Since $|M|$ is large so is $|G|$, and hence so is at least one of $q$ and $\operatorname{dim}(V)$. From Lemma 11, $\operatorname{dim}(V)$ is large compared with $s_{4}(\mathcal{M})$ and so Lemma 12 applies. Let $a \in M$ and $U \leq V$ be the subspace fixed by $\varphi\left(G_{a}\right)$. Now $g(a) \mapsto \varphi(g)(U)(g \in$ $G)$ determines the desired isomorphism of $\mathcal{M}$ and $\left(U^{P G(V)} ; G^{*}\right)$. Note that $S$ acts transitively on $M$ because $S \unlhd G$ and $G$ acts primitively. So in this case we get St. 1.

The remaining case is that in which $G(V)=\mathrm{SL}(V)$, and $\varphi$ can be extended to

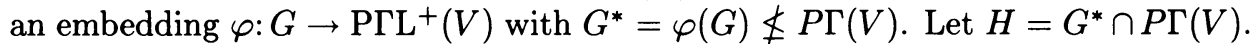
Then $\left|G^{*}: H\right|=2$ since $\left|\mathrm{P}^{+} L^{+}(V): \operatorname{P\Gamma L}(V)\right|=2$. Let $a \in M$ and $x \in \varphi\left(G_{a}\right) \backslash H$. Such $x$ exists because $G=S G_{a}$. Towards a contradiction suppose $\varphi\left(G_{a}\right) \cap H$ is maximal in $H$. By Lemma $12, \varphi\left(G_{a}\right) \cap H$ is the stabilizer in $H$ of a subspace $U$. By an argument made below we can suppose $\operatorname{dim}(U) \neq \operatorname{dim}(V) / 2$, whence $\operatorname{dim}(x(U)) \neq \operatorname{dim}(U)$. Thus we can find $y \in \varphi\left(G_{a}\right) \cap H$ such that $x^{-1} y x$ does not fix $U$. But $x^{-1} y x \in \varphi\left(G_{a}\right) \cap H$ since $H \triangleleft G^{*}$, contradiction. Therefore we have $\varphi\left(G_{a}\right) \cap H<K_{0}<H$ with $K_{0}$ maximal in $H$. Let $K_{1}=K_{0}^{x}$. Then $\varphi\left(G_{a}\right) \cap H<K_{1}<H$ because $H \triangleleft \varphi(G)$. Also, $\left|\varphi\left(G_{a}\right): \varphi\left(G_{a}\right) \cap H\right|=2$ since $\left|G^{*}: H\right|=2$. Thus $K_{1}^{x}=K_{0}$ and $K_{0} \cap K_{1}$ is $\varphi\left(G_{a}\right) \cap H$-invariant, and hence $\varphi\left(G_{a}\right)$-invariant. Thus $\left(K_{0} \cap K_{1}\right) \varphi\left(G_{a}\right)$ is a subgroup of $\varphi(G)$. Since $G$ acts primitively, $\varphi\left(G_{a}\right)$ is maximal in $\varphi(G)$, whence $\left(K_{0} \cap K_{1}\right) \varphi\left(G_{a}\right)$ is either $\varphi\left(G_{a}\right)$ or $G^{*}$. Towards a contradiction suppose $\left(K_{0} \cap K_{1}\right) \varphi\left(G_{a}\right)=G^{*}$. Then $K_{0} \cap K_{1} \triangleleft G^{*}$, so $\varphi^{-1}\left(K_{0} \cap K_{1}\right)$ acts transitively. Hence

$$
\varphi^{-1}\left(K_{0} \cap K_{1}\right) \geq \varphi^{-1}(H)
$$

since $\varphi^{-1}\left(K_{0} \cap K_{1}\right) \geq \varphi^{-1}(H) \cap G_{a}$. This implies that $K_{0} \cap K_{1} \geq H$, contradiction. It follows that $\left(K_{0} \cap K_{1}\right) \varphi\left(G_{a}\right)=\varphi\left(G_{a}\right)$. Hence $K_{0} \cap K_{1} \leq \varphi\left(G_{a}\right)$ and so $K_{0} \cap K_{1}=$ $\varphi\left(G_{a}\right) \cap H$. 
By Lemma $12, K_{0}$ is the stabilizer in $H$ of a subspace $U$ and $K_{1}$ is the stabilizer of a subspace $W$. Every automorphism of $\operatorname{PSL}(V)$ not in $\mathrm{P} \Gamma L(V)$ is induced by a semilinear transformation of $V$ onto the dual space. Thus $\operatorname{dim}(U)+\operatorname{dim}(W)=\operatorname{dim}(V)$ since $K_{0}^{x}=K_{1}$. Now $g(a) \mapsto\{\varphi(g)(U), \varphi(g)(W)\}(g \in G)$ determines an isomorphism of $\mathcal{M}$ and $\left(\{U, W\}^{\operatorname{PSL}(V)} ; G^{*}\right)$. If $U \cap W \notin\{(0), U, W\}$, then a nontrivial $\varphi(G)$-invariant equivalence relation on $\{U, W\}^{\varphi(G)}$ is defined by $\left\{U_{0}, W_{0}\right\} \sim$ $\left\{U_{1}, W_{1}\right\}$ if $U_{0} \cap W_{0}=U_{1} \cap W_{1}$ and $U_{0} \oplus W_{0}=U_{1} \oplus W_{1}$. This pulls back onto $M$, contradiction. Hence $U \cap W=(0)$ or $U \cap W=U$, where we interchange $U$ and $W$ if necessary.

Now consider $U$. Suppose $G(V)$ is $\operatorname{SL}(V)$ and $\operatorname{dim}(V)=n$. Since the action of $P \Gamma(V)$ on $k$-spaces is isomorphic to the action on $(n-k)$-spaces, by starting with a different embedding of $G$ in $\operatorname{P\Gamma L}(V)$ if necessary, we ensure that $\operatorname{dim}(U) \leq$ $\operatorname{dim}(V) / 2$. When $G(V) \neq \mathrm{SL}(V)$, each of $U \cap U^{\perp}, U \oplus V^{\perp}, U^{\perp}$,

$$
\left(V^{\perp} \oplus U\right) \cap U^{\perp} \cap\{v: Q(v)=0\}
$$

is the image of $U$ under a certain $G(V)$-invariant mapping. Either (b) holds, or by replacing $U$ by one of the above subspaces if necessary, we get (a) but with a weaker bound, namely $\operatorname{dim}(U) \leq(\operatorname{dim}(V)+1) / 2$.

Using the notation of Fact 1, suppose first that $U$ is nonsingular modulo $V^{\perp}$. Then $l \leq(k-3) / 2$. Using the elements of $G(V)$ determined by even permutations of the pairs $\left(e_{i}, f_{i}\right)$ as $X$ runs through $U^{G(V)}, \operatorname{codim}_{U}(X \cap U)$ runs through at least the integers $2 i(i \leq l)$. Therefore $s_{2}(\mathcal{M}) \geq l+1$. But $\operatorname{dim}(U) \leq 2 l+4$. Hence $\operatorname{dim}(U) \leq 2 s_{2}(\mathcal{M})+2$. If $U$ is totally isotropic (totally singular in case there is a quadratic form), then $\operatorname{dim}(U) \leq l+4$. Using the other elements of $G(V)$ mentioned in Fact $1, \operatorname{codim}_{U}(X \cap U)$ runs through at least the even integers $\leq l$. Therefore $s_{2}(\mathcal{M}) \geq(l+1) / 2$. Hence $\operatorname{dim}(U) \leq 2 s_{2}(\mathcal{M})+3$. Clearly, if $G(V)=\operatorname{SL}(V)$, a similar argument works.

Finally, we come to the bound on $q$. The idea is similar to that of Lemma 11. First consider possibility (a) for $U$. Let $G(V)$ be $\mathrm{SL}(V)$. Choose $T$ in the orbit of $U$, holding $W$ fixed in the case of St. 2, such that $\operatorname{dim}(U \cap T)=\operatorname{dim}(U)-1$. Let $X \subseteq M$ be

$$
\psi^{-1}(\{Y: U \cap T<Y<U \oplus T\})
$$

if $\mathcal{M}$ falls under St. 1 , and be defined analogously if $\mathcal{M}$ falls under St. 2. If $g \in G$ and $|X \cap g(X)|>1$ then $g(X)=X$. The permutation structure $(X, G(\{X\}))$ is isomorphic to the projective line over $F_{q}$, with group possibly augmented by some of the field automorphisms. Hence $s_{4}(\mathcal{M})>s_{1}\left(F_{q}\right)$ and we are done. If $G(V) \neq \mathrm{SL}(V)$, choose $T \leq U, u \in U \backslash T$, and $v \in U^{\perp} \backslash U$ such that $U \cap V^{\perp} \leq T$, $\operatorname{dim}(T)=\operatorname{dim}(U)-1$, and $Q(v)=0$ if a quadratic form is present. Note that for each $\alpha \in F_{q}, T \oplus\langle u+\alpha v\rangle$ is in the $G(V)$-orbit of $U$ by Witt's Theorem. Let $X$ be

$$
\psi^{-1}\left(\{Y: T<Y<T \oplus\langle u, v\rangle\} \cap U^{G(V)}\right)
$$

and proceed as before. $(X, G(\{X\}))$ is either the projective line or the projective line with a point deleted acted upon by some subgroup of $\operatorname{P\Gamma L}(q, 2)$. We finish as before.

Now we consider possibility (b) for $U$. We use Fact 5. Let $T=U \cap\left\langle e_{1}\right\rangle^{\perp}$. For each $\lambda \in F_{q}$ the linear transformation taking $f_{1}$ to $\lambda^{2} e_{1}+f_{1}+\lambda d$ and fixing all the 
other basis vectors is clearly an isometry and hence in $\Omega(V)$ since $\Omega(V)=O(V)$. Hence, if $T<Y \leq V$ and $Y \cap V^{\perp}=(0)$, then $Y \in U^{\Omega(V)}$. Letting $X$ be

$$
\psi^{-1}\left(\left\{Y: T<Y \leq V, Y \cap V^{\perp}=(0)\right\}\right)
$$

we can argue as before.

This completes the proof of the lemma.

For the alternating groups we have a stronger result:

LEMMA 14. There is a function $m: \omega \rightarrow \omega$ with the following property. Let $\mathcal{M}=(M ; G)$ be a transitive permutation structure, $|M|>m\left(s_{2}(\mathcal{M})\right), n<\omega$, and $\varphi$ be an isomorphism of $G$ onto $\operatorname{Alt}(n)$. There is a finite relational structure $₹$ with $|\mathcal{F}|<s_{2}(\mathcal{M})$ such that $\varphi$ can be extended to an isomorphism $\varphi: \mathcal{M} \rightarrow \mathcal{N}=$ $(N ; \operatorname{Alt}(n))$, where $N$ is the set of all copies of $\mp$ with universe $\subseteq n$.

Proof. Let $a \in M$ and $H$ denote $\operatorname{Alt}(n)$. We shall suppose that $|M|$ is large compared with $s_{2}(\mathcal{M})$ and find the desired extension of $\varphi$. Since $|M|$ is large, so is $|G|$ and hence so is $n$. Thus $G$ is simple. Let $E$ be a maximal $G$-invariant equivalence relation on $M$. Let $C=a / E$ and $\mathcal{M}^{*}=\left(M^{*} ; G^{*}\right)$ denote $\mathcal{M} / E$. Since $G^{*}$ is a nontrivial homomorphic image of $G, G^{*} \cong G$. Since $E$ is maximal, $\mathcal{M}^{*}$ is primitive. Hence $\varphi(G(\{C\}))$ is a maximal subgroup of $H$. Also, $s_{2}\left(\mathcal{M}^{*}\right) \leq s_{2}(\mathcal{M})$ and so $n$ is large compared with $s_{2}\left(\mathcal{M}^{*}\right)$. From Bannai $[4$, Theorem 1.3] $\varphi(G(\{C\}))=H(\{A\})$ for some $A \subseteq n$ such that $|A|=s_{2}\left(\mathcal{M}^{*}\right)-1$. Clearly, $\varphi\left(G_{a}\right) \leq H(\{A\})$.

If $H(A) \leq \varphi\left(G_{a}\right)$, we are done. Otherwise, replace $\operatorname{Alt}(n)$ by $\operatorname{Alt}(n \backslash A), M$ by $M^{\#}=a^{\varphi^{-1}(H(A))}$ and $G$ by the group $G^{\#} \leq \operatorname{Sym}\left(M^{\#}\right)$ induced by $\varphi^{-1}(H(A))$. Clearly $s_{2}\left(\mathcal{M}^{\#}\right) \leq|A| ! s_{2}(\mathcal{M})$, whence $s_{2}\left(\mathcal{M}^{\#}\right)$ is bounded in terms of $s_{2}(\mathcal{M})$. Repeating the argument of the last paragraph we find $B \subseteq n \backslash A$ such that $|B|$ is bounded in terms of $s_{2}(\mathcal{M})$ and $\varphi\left(G_{a}\right) \cap H(A) \leq H(\{B\})$. Since $|H(\{A\}): H(A)|$ is bounded in terms of $s_{2}(\mathcal{M})$ we can actually find $B$ above such that $\varphi\left(G_{a}\right) \leq$ $H(\{B\})$. Hence $\varphi\left(G_{a}\right) \leq H(\{A \cup B\})$.

In this way we can find larger and larger sets $D \subseteq n$ such that $\varphi\left(G_{a}\right) \leq H(\{D\})$. For each set $D$ we can argue that $|D|<s_{2}(\mathcal{M})$. Thus eventually we find $F \subseteq n$ such that $|F|<s_{2}(\mathcal{M})$ and $H(F) \leq \varphi\left(G_{a}\right) \leq H(\{F\})$. Let $\mathcal{F}$ be a relational system with universe $F$ and distinguished relations chosen so that $\operatorname{Aut}(\mathcal{F}) \leq \operatorname{Sym}(F)$ is the group induced by $G_{a}$. The rest is clear.

LEMMA 15. There is a function $m: \omega \rightarrow \omega$ with the following property. Let $\mathcal{M}=(M ; G)$ be a primitive structure, $\operatorname{Soc}(G)$ be a direct product of alternating groups, and $|M|>m\left(s_{2}(\mathcal{M})\right)$. Then $\mathcal{M}$ is coordinatizable.

Proof. We shall suppose $|M|$ is large compared to $s_{2}(\mathcal{M})$. Thus $|G|$ is also large. From the O'Nan-Scott lemma and the remarks following it,

$$
N={ }_{\mathrm{dfn}} \operatorname{Soc}(G)=\prod_{i \in I} S_{i} \quad \text { (direct product) }
$$

where the $S_{i}$ are isomorphic alternating groups. For $a \in M, N_{a}=N \cap G_{a}=$ $\prod_{i \in I} S_{i a}$ where $(1)<S_{i a}={ }_{\mathrm{dfn}} S_{i} \cap G_{a}<S_{i}$. Also $|I|<s_{2}(\mathcal{M})$. Let $\varphi_{i}: S_{i} \rightarrow \operatorname{Alt}(n)$ be isomorphisms. Then $n$ is large compared with $s_{2}(\mathcal{M})$.

Let $A_{j}$ denote the stabilizer of $j$ in $\operatorname{Alt}(n)$ and $S_{i, j}=\varphi_{i}^{-1}\left(A_{j}\right)(i \in I, j<n)$. Let $H_{i}=\left\{S_{i, j}: j<n\right\}$. Then $S_{i}$ acts on $H_{i}$ by conjugation by the inverse. Let 
$H=\bigcup_{i \in I} H_{i}$. Then $G$ acts on $H$ by conjugation by the inverse, because any automorphism of $\operatorname{Alt}(n)$ is induced by $\pi \in \operatorname{Sym}(n)$ and so permutes $\left\{A_{j}: j<n\right\}$. The coordinate system we need is $\left(H ; G^{*}\right)$, where $G^{*} \leq \operatorname{Sym}(H)$ is the group induced by $G$. It is clear that the $H_{i}$ are the classes of a $G^{*}$-invariant equivalence relation, since $G$ permutes the $H_{i}$. For the rest it is sufficient to exhibit a $G$ invariant map $a \mapsto F_{a}(a \in M)$ such that $F_{a} \subseteq H$ and $\left|F_{a}\right|<s_{2}(\mathcal{M})$. Note that $G$ permutes the $H_{i}$ transitively, because $\mathcal{M}$ is primitive.

Let us call sets "small" if their size is bounded in terms of $s_{2}(\mathcal{M})$. Fix $b \in M$. From Lemma 9 there is a primitive homogeneous substructure $\left(b^{S_{i}} ; K_{i}\right)$ of $\mathcal{M}$, with $\operatorname{Soc}\left(K_{i}\right)$ the restriction of $S_{i}$ to $b^{S_{i}}$, and with some abuse of notation we can write $S_{i} \leq K_{i} \leq \operatorname{Aut}\left(S_{i}\right)$. From Lemma 14 there is a small set $X_{i b} \subseteq n$ such that $\varphi_{i}\left(S_{i b}\right)$ is the set stabilizer of $X_{i b}$ in $\operatorname{Alt}(n)$. Let $F_{i b}=\varphi_{i}^{-1}\left(\left\{A_{j}: j \in X_{i b}\right\}\right)$. Then $S_{i b}$ fixes $F_{i b}$ setwise in its action on $H_{i}$. Define $F_{a}=\bigcup_{i \in I} F_{i a}$. From Remark 2 following Lemma 8, $a \mapsto\left\{S_{i a}: i \in I\right\}(a \in M)$ is a $G$-invariant map. Hence $a \mapsto F_{a}(a \in M)$ is $G$-invariant. As $a_{0}, a_{1}$ vary in $M,\left|F_{a_{0}} \cap F_{a_{1}}\right|$ takes all values $\leq\left|F_{b}\right|$. Thus $s_{2}(\mathcal{M})>\left|F_{b}\right|$. This completes the proof.

4. Proof of Theorem 2. Here we will prove Theorem 2 using Lemmas A and $\mathrm{B}$ from $\S 2.1$ together with the facts about permutation structures assembled in $\S 3$.

4.1. The setting. Recall the discussion from $\S 2.1$. It is assumed that Theorem 2 holds for structures of height at most $h$. In this section we complete the induction step by showing that the theorem holds for structures of height $h+1$. Let $\mathcal{M} \in$ $\operatorname{Hom}(L, \omega)$ be transitive, $\operatorname{ht}(\mathcal{M})=h+1$, and $E$ be a maximal 0 -definable equivalence relation on $M$. We have to show that either $|M / E|$ is bounded in terms of $L$ or $\mathcal{M} / E$ is coordinatizable. As remarked in $\S 2.1$ we can suppose that $|M|$ is finite. Let $l$ denote the arity of $L$.

Let $\mathcal{M}=(M ; G)$ as a permutation structure and $\mathcal{N}=(N, K)$ denote $\mathcal{M} / E$. Then $\mathcal{N}$ is primitive, so we can apply Lemma 8 and the theory associated with it. There are two cases according as $\operatorname{Soc}(K)$ is abelian or not.

4.2. The case in which $\operatorname{Soc}(K)$ is abelian. Let $\operatorname{Soc}(K)=A$ be abelian. Let $b \in N$ and $H=K_{b}$. Then $K=A H$ and by Lemma 10 we can identify $N$ with $A$ (and in particular $b$ with $O \in A$ ) so that $A$ acts by translation and $H$ by conjugation by the inverse. The crucial point is that once $b$ is fixed $\mathcal{N}$ has at least the structure of the group $A$. Below we shall show that this conflicts with the homogeneity of $\mathcal{M}$.

Let $s(i)=s_{(l+1) i}(\mathcal{M}), m(i)=\max (2 i, l+1)+s(i)$, and $F(i)=2^{m(i)}$. By Lemma A we can find $e$ bounded in terms of $L$ such that $<e$.

(**) Any $₹ \subseteq M / E$ of cardinality $F(e)$ has a replacement $\mathcal{F}^{\prime}$ in $\mathcal{M}$ by sets of size

Now apply Lemma $B$ to $\mathcal{N}$. Let $m=m(e)=\max (2 e, l+1)+s(3)$. If $|M / E|$ is large enough, then we have an indiscernible set $I=\left\{b_{0}, b_{1}, \ldots, b_{m}\right\}$ of size $m+1$ in $\mathcal{N}$. We can suppose $b_{0}=b$ the element identified with $0 \in A$. We see the $b_{i}$ 's as $E$-classes. Let $a_{i} \in A$ be the element identified with $b_{i} \in N$. Let $₹$ consist of all $x \in N$ such that the corresponding element of $A$ is the sum of some subset of $\left\{a_{i}: i \leq m\right\}$. Note that $|\mathcal{F}|=F(e)$. Let $\mathcal{F}^{\prime}$ be a replacement for $\mathcal{F}$ in $\mathcal{M}$ by sets of size $<e$. The element of $\mathcal{F}^{\prime}$ contained in $b_{i}$ will be denoted $b_{i}^{\prime}$. Set $s=s(e)$. For $0<i \leq s$ let $i^{*}$ denote $\max (2 e, l+1)+i$ and let $c_{i} \in N$ be the $E$-class identified with $\sum\left\{a_{h}: h \leq i^{*}\right\}$ taken in $A$. The element of $\mathcal{F}^{\prime}$ contained in $c_{i}$ is denoted $c_{i}^{\prime}$. 
All the permutations of $\mathcal{F}^{\prime}$ induced by those permutations of $\mathcal{F}$ which fix $b_{0}$, permute $I \backslash\left\{b_{0}\right\}$ arbitrarily, and are extended to $\mathcal{F} \backslash I$ naturally, are in fact induced by $G$ since $\mathcal{F}^{\prime}$ is a replacement for $\mathcal{F}$ and $I$ is a set of indiscernibles.

Fix $j, k, 1 \leq j<k \leq s$, and consider the group $S \leq G$ consisting of all $g \in G$ leaving the following subsets of $M$ invariant:

$$
b_{0}^{\prime}, c_{j}^{\prime}, c_{k}^{\prime}, b_{i}^{\prime} \quad\left(j^{*}<i \leq k^{*}\right), \quad \bigcup\left\{b_{i}^{\prime}: i \leq j^{*}\right\} .
$$

Let $I^{\prime}$ denote $\left\{b_{i}^{\prime}: 1 \leq i \leq j^{*}\right\}$. As the elements of $I^{\prime}$ are separated by $E$-classes, the elements of $S$ induce permutations of $I^{\prime}$ and conversely every permutation of $I^{\prime}$ is induced by some $g \in S$. Thus we have a surjective homomorphism: $S \rightarrow \operatorname{Sym}\left(I^{\prime}\right)$. We also have a natural homomorphism: $S \rightarrow \operatorname{Sym}\left(c_{j}^{\prime} \cup c_{k}^{\prime}\right)$ with kernel $T$ say. Since $\left|c_{j}^{\prime} \cup c_{k}^{\prime}\right|<2 e,|S: T|<(2 e)$ !. Let $U$ be the image of $T$ in $\operatorname{Sym}\left(I^{\prime}\right)$. Then $U \triangleleft \operatorname{Sym}\left(I^{\prime}\right)$. If $U=(1)$, then $|S: T| \geq\left|\operatorname{Sym}\left(I^{\prime}\right)\right| \geq(2 e)$ !, contradiction. Therefore $U \geq \operatorname{Alt}\left(I^{\prime}\right)$.

For each $i, 1 \leq i \leq s$, choose $d_{i} \in c_{i}^{\prime}$. Now $s=s(e)=s_{(l+1) e}(\mathcal{M})$ is greater than the number of 1-types over $C=\bigcup\left\{b_{i}^{\prime}: i \leq l\right\}$ which are realized in $\mathcal{M}$. Therefore we can choose $j, k$ such that $1 \leq j<k \leq s$ and $\operatorname{tp}\left(d_{j} \mid C\right)=\operatorname{tp}\left(d_{k} \mid C\right)$. Let $D=\bigcup\left\{b_{i}^{\prime}: i \leq j^{*}\right\}$. Any $l$-tuple in $D$ can be mapped into $C$ by $g \in T$ which fixes $d_{j}$ and $d_{k}$. Since $\mathcal{M}$ is $l$-ary, $\operatorname{tp}\left(d_{j} \mid D\right)=\operatorname{tp}\left(d_{k} \mid D\right)$. It follows that there exists $g \in K$ such that $g\left(c_{j}\right)=c_{k}$ and $g\left(b_{i}\right)=b_{i}\left(i \leq j^{*}\right)$. Via the identification of $N$ and $A, g$ becomes an automorphism of $A$ fixing $a_{i}\left(1 \leq i \leq j^{*}\right)$ but moving $\sum\left\{a_{i}: 1 \leq i \leq j^{*}\right\}$. This contradiction completes the treatment of this case.

4.3. The case in which $\operatorname{Soc}(K)$ is nonabelian. Recall that, since $\mathcal{N}=\mathcal{M} / E$ is primitive, $\operatorname{Soc}(K)$ is a direct product of isomorphic simple groups.

From Lemma 15, if the factors of $\operatorname{Soc}(K)$ are alternating groups, then either $\mathcal{N}$ is coordinatizable or $|N|$ is bounded in terms of $s_{2}(\mathcal{N})$ and hence in terms of $L$. Below we assume that the factors of $\operatorname{Soc}(K)$ are not alternating groups.

From Lemma 9 we may suppose that $\operatorname{Soc}(K)$ is simple, passing if necessary to a primitive homogeneous substructure.

From Lemma 11, since $s_{4}(\mathcal{N})$ is bounded in terms of $L$, either $|N|$ is bounded in terms of $L$ or $\operatorname{Soc}(K)$ is a classical group, over $F_{q}$ say, of large rank, i.e., the associated vector space has large dimension.

Applying Lemma 13 there are two broad possibilities for the isomorphism type of $\mathcal{N}$, namely St. 1 and St. 2. We will treat only the former; the argument can easily be adapted to St. 2 . So there is a vector space $V$ over $F_{q}$ with $q$ bounded in terms of $L$ and $\operatorname{dim}(V)$ large, $G(V)$ is a classical group, and there is an isomorphism $\psi: \mathcal{N} \rightarrow\left(U^{P G(V)} ; G^{*}\right)$, where $P G(V) \leq G^{*} \leq P \Gamma(V)$ and $U$ is a subspace of $V$.

We obtain a contradiction using the same idea as in $\S 4.1$. Let $p$ be the characteristic of $F_{q}, s(i)=s_{l i}(\mathcal{M}), m(i)=[\max (2 i, l+1)+s(i)] p+1$, and $F(i)$ denote the number of subsets of $\{1, \ldots, m(i)\}$ whose size is congruent to $1 \bmod p$. From Lemma A of $\S 2.1$ we can find $e$ bounded in terms of $L$ such that

$(* *)$ Any $₹ \subseteq M / E$ of cardinality $F(e)$ has a replacement $\mathcal{F}^{\prime}$ in $\mathcal{M}$ by sets of size $<e$.

For $1 \leq i \leq s==_{\mathrm{dfn}} s(e)$, let $i^{*}=[\max (2 e, l+1)+i] p+1$.

Consider first the case in which $V^{\perp}=(0)$. From Lemma $13 \operatorname{dim}(U) \leq 2 s_{2}(\mathcal{N})+3$ is bounded in terms of $L$. Let $m$ denote $s^{*}=m(e)$. Since $\operatorname{dim}(V)$ is large we can find $U_{1}, \ldots, U_{m} \in U^{P G(V)}$ pairwise orthogonal (this requirement is vacuous if $G(V)$ 
is $\mathrm{SL}(V))$ and mutually linearly independent, i.e.

$$
U_{i} \cap \bigoplus \sum\left\{U_{j}: 1 \leq j \leq m, j \neq i\right\}=(0) \quad(1 \leq i \leq m)
$$

Choose a family $\tau$ of compatible isometries between the $U_{i}$ 's. Let $\theta$ denote the set of all $\theta \subseteq\{1, \ldots, m\}$ such that $|\theta| \equiv 1(\bmod p)$. For each $\theta \in \Theta$ let $V_{\theta}$ be the diagonal (with respect to $\tau$ ) subspace inside $\bigoplus \sum\left\{U_{i}: i \in \theta\right\}$. Since $|\theta| \equiv 1(\bmod p)$, $V_{\theta}$ and $U$ are isometric in $V$. By Witt's Theorem $V_{\theta} \in U^{P G(V)}$. By the same token, if $\pi \in \operatorname{Sym}(\{1, \ldots, m\})$, there exists $g \in P G(V)$ such that $g\left(V_{\theta}\right)=V_{\pi(\theta)}(\theta \in \Theta)$. Let $\mathcal{F}=\left\{\psi^{-1}\left(V_{\theta}\right): \theta \in \Theta\right\}$. Then $|\mathcal{F}|=F(e)$. By $(* *)$ choose $\mathcal{F}^{\prime}$ a replacement for $\mathcal{F}$ in $\mathcal{M}$ by sets of size $<e$.

Let $b_{i}^{\prime}$ be the element of $\xi^{\prime}$ contained in $\psi^{-1}\left(U_{i}\right), c_{j}^{\prime}$ be the element of $\xi^{\prime}$ contained in $\psi^{-1}\left(V_{\left\{1, \ldots, i^{*}\right\}}\right)$ and $d_{i} \in c_{i}^{\prime}$ be arbitrary. Arguing exactly as in the case of an abelian socle, we can show that there exist $j, k$ such that $1 \leq j<k \leq m$ and $\operatorname{tp}\left(d_{j} \mid D\right)=\operatorname{tp}\left(d_{k} \mid D\right)$, where $D=\bigcup\left\{b_{i}^{\prime}: 1 \leq i \leq j^{*}\right\}$. In $\left(U^{P \bar{G}(V)} ; G^{*}\right)$ this means that $V_{\left\{1, \ldots, j^{*}\right\}}$ can be moved to $V_{\left\{1, \ldots, k^{*}\right\}}$ while holding $U_{1}, \ldots, U_{j^{*}}$ fixed as subspaces. This is absurd, because $V_{\left\{1, \ldots, j^{*}\right\}}$ is in the linear span of $U_{1}, \ldots, U_{j^{*}}$ but $V_{\left\{1, \ldots, k^{*}\right\}}$ is not.

This leaves us the case in which $V^{\perp} \neq(0)$. Now $q$ is even, $\operatorname{dim}(V)$ is odd, and $G(V)$ is $O(V)=\Omega(V)$. If $\operatorname{dim}(U)<\operatorname{dim}(V)-1$ or $V^{\perp} \leq U$, then by Lemma $13 \operatorname{dim}(U) \leq 2 s_{2}(N)+3$, and either $U$ is nonsingular modulo $V^{\perp}$, or $U$ is totally singular. In the totally singular case we follow the previous line exactly. If $U$ is nonsingular modulo $V^{\perp}$, we choose nonsingular $T<U$ with codimension 1 in $U$. We use $T$ rather than $U$ to generate $U_{1}, \ldots, U_{m}$. Otherwise everything goes as before. Finally suppose now that $\operatorname{dim}(U)=\operatorname{dim}(V)-1$ and $V^{\perp} \cap U=(0)$. Again we use Fact 5 from $\S 3.3$. The key point is that the linear transformation $\tau_{i}$ which takes $e_{i}$ to $e_{i}+f_{i}+d$ and fixes all the other basis vectors belongs to $O(V)$. We define $U_{i}=\tau_{i}(U)(1 \leq i \leq m)$. The $\tau_{i}$ commute with each other and so for $\theta \in \Theta$ we define $\tau_{\theta}=\prod\left\{\tau_{i}: i \in \theta\right\}$ and $V_{\theta}=\tau_{\theta}(U)(\theta \in \Theta)$. Arguing as in the previous case we see that there are numbers $j, k, 1 \leq j<k \leq m$, and $g \in G^{*}$ such that $g$ moves $V_{\left\{1, \ldots, j^{*}\right\}}$ to $V_{\left\{1, \ldots, k^{*}\right\}}$ and fixes $U_{1}, \ldots, U_{j^{*}}$. Now

$$
U=\left(U_{2} \cap U_{3}\right) \oplus\left(U_{3} \cap U_{1}\right) \oplus\left(U_{1} \cap U_{2}\right),
$$

whence $g$ fixes $U$ because $m(j) \geq 3$. It is easy to check that

$$
U \cap\left(U \cap U_{i}\right)^{\perp}=\left\langle f_{i}\right\rangle \quad(1 \leq i \leq m)
$$

and

$$
U \cap\left(U \cap V_{\theta}\right)^{\perp}=\left\langle\sum\left\{f_{i}: i \in \theta\right\}\right\rangle \quad(\theta \in \Theta) .
$$

Hence $g$ fixes $\left\langle f_{i}\right\rangle(1 \leq i \leq m(j))$ but moves $\left\langle f_{1}+\cdots+f_{m(j)}\right\rangle$ to $\left\langle f_{1}+\cdots+f_{m(k)}\right\rangle$. This contradiction completes the proof of Theorem 2 .

5. Existence of indiscernibles in finite structures. In this section we prove

LEMMA B. There is a function $m: \omega^{2} \rightarrow \omega$ with the following property. If $\mathcal{M}$ is finite and $|M|>m\left(r, s_{5}(\mathcal{M})\right)$, then $M$ contains a set of indiscernible of size $r$.

We are grateful to Bill Kantor for simplifying the proof. In particular, Lemma 20 of $\S 5.2$ and Steps 4 and 5 of $\S 5.3$ were entirely his idea. 
We do not know whether Lemma $B$ is true with $s_{4}(\mathcal{M})$ instead of $s_{5}(\mathcal{M})$. The projective lines over $F_{q}$ with $q=p^{n}, p$ fixed, and $n \rightarrow \infty$, show that $s_{5}(\mathcal{M})$ cannot be replaced by $s_{3}(\mathcal{M})$.

5.1. Plan of the proof and preliminaries. The proof of Lemma B has two parts. In the first part $(\S 5.2)$ we prove Lemma 20 which is a strengthening of Lemma B in that $s_{4}(\mathcal{M})$ replaces $s_{5}(\mathcal{M})$, but a special case in that the class of structures is severely restricted. In the second part (§5.3), aiming for a contradiction, we study the properties of a supposed counterexample to Lemma B which has $s_{5}(\mathcal{M})$ as small as possible.

By a counterexample to Theorem 1 we mean a triple $\langle r, s, \mathbf{M}\rangle$ satisfying

(i) $r, s<\omega$,

(ii) $\mathbf{M}$ is a countably infinite family of finite structures no two of the same cardinality,

(iii) $s_{5}(\mathcal{M})=s$ for all $\mathcal{M} \in \mathbf{M}$,

(iv) no $\mathcal{M} \in \mathbf{M}$ contains an indiscernible set of size $r$.

Speaking loosely we often refer to a typical member $\mathcal{M}$ of $\mathbf{M}$ as the counterexample. If we say the counterexample has some property of structures we mean that all but a finite number of members of $\mathbf{M}$ have the property.

The rest of this section consists of four lemmas required for the proof which follows.

LEMMA 16. Let $r>\max (5, e), \mathcal{M}=(M ; G)$ be a permutation structure, and $E$ be a $G$-invariant equivalence relation on $M$. Let each $E$-class have size $e,|M / E|>$ $(r-1)\left(\log _{2} e+1\right)$, and $M / E$ be quasi-indiscernible. Then $M$ contains a quasiindiscernible set of size $r$ of pairwise E-equivalent elements.

PROOF. If $e=1$, the conclusion is immediate. So suppose $e \geq 2$. If the subgroup $G(U)$ which fixes the union $U$ of $(r-1) E$-classes pointwise is transitive on a further $E$-class $C$, then any $r E$-inequivalent elements of $M$ are quasi-indiscernible. Thus below we may assume that $G(U)$ is intransitive on $C$.

Let $H \leq G$ be the subgroup $G(U / E)$ fixing $U / E$ pointwise. $G(U) \unlhd H$ and the natural map $\varphi: H \rightarrow \operatorname{Sym}(M \backslash U) / E$ has range $\geq \operatorname{Alt}((M \backslash U) / E)$. Hence there are two possibilities:

(i) $\varphi(G(U))=(1)$.

(ii) $\varphi(G(U)) \geq \operatorname{Alt}((M \backslash U) / E)$.

In case (i), there is a natural surjection: $\bar{H} \rightarrow \varphi(H)$, where $\bar{H} \leq \operatorname{Sym}(U)$ is the group induced by $H$. Now $\bar{H}$ is isomorphic to a subgroup of $\operatorname{Sym}(\{1,2, \ldots, e\})^{r}$ (direct product) and so all its composition factors have order $\leq|\operatorname{Alt}(e)|$. But $\varphi(H)$ has $\operatorname{Alt}((M \backslash U) / E)$ as a composition factor. This contradicts the existence of the surjection since

$$
|(M \backslash U) / E|>(r-1) \log _{2} e \geq \max (5, e) .
$$

In case (ii) $(M \backslash U) / E$ is quasi-indiscernible under $G(U)$. Choose $x \in C$ such that $\left|x^{G(U)} \cap C\right| \leq e / 2$. We finish by induction on $e$ applied to the permutation structure $\left(x^{G(U)} ; G(U)\right)$.

LEMMA 17. Let $S$ be a group, $S^{\prime}=S$, and $S / Z(S)$ be isomorphic to Alt $(n)$ or a classical simple group $P G(V)$ over $F_{q}$ with $\operatorname{dim}(V)=n$. Let $S \leq \mathrm{GL}(A)$ where 
$A$ is a vector space over $F_{p}, p$ prime, of dimension $k$. Then

(i) $k \geq n / 30$, and

(ii) $k \geq q^{n / 30}$ in case $S / Z(S) \cong P G(V)$ and $p \nmid q$.

PROOF. (i) By induction we can show that, if $S / Z(S) \cong \operatorname{Alt}(n)$, then $k \geq n / 10$. We start at $n=10$ and use a subgroup $\operatorname{Alt}(m) \times \operatorname{Alt}(m)$ or $\operatorname{Alt}(m) \times \operatorname{Alt}(m+1)$ when $n=2 m$ or $2 m+1$ respectively. For the rest, note that $P G(V)$ has a subgroup isomorphic to $\operatorname{Alt}([n / 3]+1)$.

(ii) This is a result of Landazuri and Seitz [16, p. 419].

LEMMA 18. Let $S$ be a finite simple group of Lie type over $F_{q}$ and $p$ be the characteristic of $F_{q}$. Let $U$ be a Sylow p-subgroup of $S$ and $U \leq L \leq S$. There exists a parabolic subgroup $P$ of $S$ such that $L \unlhd P$ and $P / L$ is abelian.

PROOF. This is a result of Tits, see Seitz [18, Lemma 1.6]. We use the notation of Carter [6]. Since any two Sylow $p$-subgroups are conjugate we may suppose that $U$ is the unipotent subgroup discussed in $[6, \S 5]$. Below for convenience of notation we discuss the untwisted case; the proof in the twisted case is analogous. Choose a minimal set $J$ of fundamental roots such that $L \leq P_{J}=B N_{J} B$. The argument of Seitz (loc. cit.) gives

$$
\left\langle U^{P_{J}}\right\rangle=\left\langle U, n_{r}: r \in J\right\rangle \leq L,
$$

where $\left\langle U^{P_{J}}\right\rangle$ denotes the group $K$ generated by all the conjugates of $U$ in $P_{J}$. Clearly $K \unlhd P_{J}$. Also, we have $B=H U, P_{J}=B N_{J} B$, and $N_{J}=H\left\langle n_{r}: r \in J\right\rangle$. Further, $H \triangleleft N$ and $H$ normalizes $U$ because it normalizes all the root groups. Therefore

$$
\begin{aligned}
P_{J} & =B N_{J} B=U H H\left\langle n_{r}: r \in J\right\rangle U H \\
& =H U\left\langle n_{r}: r \in J\right\rangle U \leq H K .
\end{aligned}
$$

Thus $P_{J}=H K$, whence $P_{J} / K$ is a quotient of $H$ and so abelian. Since $K \leq L \leq$ $P_{J}, L \unlhd P_{J}$ and $P_{J} / L$ is also abelian.

LEMMA 19. Let $P G(V)$ be one of the classical simple groups discussed in $\S 3.2$ and $P \leq P G(V)$ be a parabolic subgroup of $P G(V)$. Then there are proper subspaces $U_{1}<U_{2}<\cdots<U_{j}$ (except that if $G(V)=\Omega^{+}(V)$ and $\operatorname{dim}\left(U_{j}\right)=\operatorname{dim}(V) / 2$ the sequence may end $U_{j-2}<U_{j-1}, U_{j}$ with $\operatorname{dim}\left(U_{j}\right)=\operatorname{dim}\left(U_{j-1}\right)$ and $U_{j-2}=$ $\left.U_{j} \cap U_{j-1}\right)$ of $V$ such that $P$ is the stabilizer of $\left\{U_{1}, \ldots, U_{j}\right\}$. Further, if there is a scalar product, then the $U_{i}$ are totally isotropic, and totally singular if there is a quadratic form.

This is well known but we cannot give an explicit reference. The essential idea for a proof can be found in $[8, \mathrm{pp} .8,9]$.

5.2. A special case of Lemma $\mathrm{B}$. The case we have in mind is

LEMMA 20. There is a function $m(r, t)$ with the following property. Let $W=$ $(W ; S)$ be a permutation structure, where $W$ is a vector space over $F_{p}$, with $p$ prime, and $S \leq \mathrm{GL}(W)$ acts irreducibly on $W$. Suppose $S=S^{\prime}$ and that $S / Z(S)$ is simple. If $|W|>m\left(r, s_{4}(W)\right)$, then $W$ contains a set of indiscernibles of size $r$.

ProOF. Let $W=(W ; S)$ be as in the statement of the lemma, $\operatorname{dim}(W)=m$, and $|W|$ be very large compared with $r$ and $t=s_{4}(W)$.

Each $g \in Z(S)$ is an $S$-invariant subspace of $W^{2}$, whence $|Z(S)|<2^{t}$. Also $\bigcup\{g: g \in Z(S)\}=E_{Z}$ is an $S$-invariant equivalence relation on $W$. Let $Q$ be the 
largest orbit of $S$ on $W$ and $E \geq E_{Z} \cap Q^{2}$ be a maximal $S$-invariant equivalence relation on $Q$. Let $\mathcal{Q}=(Q / E ; T)$, where $T$ is the group induced by $S$. Then $\mathcal{Q}$ is primitive and $T$ is isomorphic to a quotient of $S$. Hence $T \cong S / Z(S)$. Also $s_{4}(\mathcal{Q}) \leq t$.

By Lemma $11 T$ is either an alternating group or a classical group $P G(V)$ over $F_{q}$ (as in §3) with $q$ bounded in terms of $t$ by Lemma 13. Let $n$ be the degree of the alternating group or $\operatorname{dim}(V)$ as the case may be. There are now three cases.

Case 1. $S / Z(S) \cong \operatorname{Alt}(n)$. Let $R=\left(Q / E_{Z} ; T_{Z}\right)$, where $T_{Z}$ is the group induced by $S$. Clearly $T_{Z} \cong \operatorname{Alt}(n)$ since $T_{Z} \cong S / Z(S)$. By Lemma $14 R$ contains a set of indiscernibles large compared with $r$ and $t$. Since each $E_{Z}$-class has size $<2^{t}, \mathcal{W}$ contains a set of indiscernibles of size $r$ by Lemma 16 .

Case 2. $S / Z(S)$ is isomorphic to a classical group $P G(V)$ over $F_{q}, \operatorname{dim}(V)=n$, and $p \nmid$ $\backslash$. By Lemma $17 m=\operatorname{dim}(W) \geq q^{n / 30}$. Now

$$
t \geq s_{4}(\mathcal{W})>s_{1}(\mathcal{W}) \geq \frac{|W|}{|S|}=\frac{|W|}{|Z(S)||S / Z(S)|}>\frac{p^{q^{n / 30}}}{2^{t} \cdot q^{n^{2}}}
$$

Thus $t 2^{t} \cdot q^{n^{2}}>2^{2^{n / 30}}$. Recall that $q$ is bounded in terms of $t$. Since $|W|$ is very large compared with $t$, so is $\left|Q / E_{Z}\right|$, and hence so is $n$. This contradicts the inequality, so this case cannot occur.

Case 3. $S / Z(S)$ is isomorphic to a classical group $P G(V)$ over $F_{q}, \operatorname{dim}(V)=n$, and $p \mid q$. Redefine $Q$ to be an orbit of $S$ on $W \backslash\{0\}$ such that $|Q|$ is prime to $p$. The span of $Q$ is an $S$-invariant subspace, and hence is $W$, since $S$ acts irreducibly. Since $|W|$ is very large, $s_{1}(\mathcal{W})<t,|Z(S)|<2^{t}$, it follows that $|S / Z(S)|$ is very large. Since $q$ is bounded in terms of $t$, so is $p$. Therefore $m=\operatorname{dim}(W)$ is very large, whence $|Q|$ is very large compared with $r$ and $t$. The same is clearly true of $n$.

Let $R=\left(Q / E_{Z} ; T_{Z}\right)$ be defined as in Case 1. Plainly there exists an isomorphism $\varphi$ of $T_{Z}$ onto $P G(V)$. Let $U \leq T_{Z}$ be a Sylow $p$-subgroup. Every orbit of $U$ on $Q / E_{Z}$ has order a power of $p$. Since $p \nmid\left|Q / E_{Z}\right|$ there exists $a \in Q / E_{Z}$ such that $U \leq L=\mathrm{dfn}\left(T_{Z}\right)_{a}$. By Lemma 18 there is a parabolic subgroup $P$ of $T_{Z}$ such that $L \unlhd P$ and $P / L$ is abelian.

For $x, y \in Q / E_{Z}$ let $x E_{1} y$ if $(x, y)=(g(a), g h(a))$ for some $g \in T_{Z}$ and $h \in P$. Then $E_{1}$ is a $T_{Z}$-invariant equivalence relation on $Q / E_{Z}$. Let $C$ denote the $E_{1}$-class of $a$ and $C=(C ; K)$, where $K \leq \operatorname{Sym}(C)$ is the group induced by $P=T_{Z}(\{C\})$. Since $L \unlhd P, K$ is naturally isomorphic to a quotient of $P / L$ and hence abelian. Since $C$ is transitive, $K$ acts regularly on $C$, i.e., $s_{2}(C)=|C|$. But $s_{2}(C)<t$ is inherited from $\mathcal{W}$. Therefore $|C|<t$.

Let $E_{P}$ be the equivalence relation on $Q$ induced by $E_{1}$, and $T_{P} \leq \operatorname{Sym}\left(Q / E_{P}\right)$ be the group induced by $S$. Let $S=\left(Q / E_{P} ; T_{P}\right)$. We have seen that the $E_{P}$-classes have size $<t 2^{t}$. By Lemma 16 it is sufficient to find a large set of indiscernibles in S.

Since $\varphi(P)$ is a parabolic subgroup of $P G(V), \varphi(P)$ is the stabilizer of a collection $₹=\left\{U_{1}, \ldots, U_{j}\right\}$ of proper subspaces of $V$ of the kind described in Lemma 19. Hence $\varphi$ extends to an isomorphism of $S$ and $\left(\mathcal{F}^{P G(V)} ; P G(V)\right)$. Let $G(V) \neq$ $\mathrm{SL}(V)$. We apply a slight refinement of Fact 1 of $\S 3.2$ which says that, if $U \neq$ $(0)$, then $e_{1}, f_{1}, \ldots, e_{k}, f_{k}$ may be chosen such that $e_{1} \in U$ as well satisfying all the other requirements. Take $U=U_{j}$. We first argue as in Lemma 13 that 
$\operatorname{dim}\left(U_{j}\right)<2 s_{2}(S)+3<2 t+3$. Hence $\mp$ is a chain. Now $U_{j} \cap W=\left\langle e_{1}, \ldots, e_{l}\right\rangle$. Let $G_{1} \leq G(V)$ be the copy of $\operatorname{Alt}(k-l)$ induced by the even permutations of the pairs $\left(e_{l}, f_{l}\right), \ldots,\left(e_{k}, f_{k}\right)$. Then $\left|f^{G_{1}}\right|=k-l+1$ and $G_{1}$ has the natural action of $\operatorname{Alt}(k-l)$ on $\mathcal{F}^{G_{1}}$. Thus in $S$ we get a set of indiscernibles of size $k-l-1$. Since $2 k \geq \operatorname{dim}(V)-4$ is large compared with $r$ and $t$, and $l$ is bounded, we have the indiscernibles we need. If $G(V)=\mathrm{SL}(V)$ the situation is slightly different. For each $i, 1 \leq i \leq j$, we have either $\operatorname{dim}\left(U_{i}\right)$ or $\operatorname{codim}\left(U_{i}\right)<s_{2}(S)<t$. By passing to the dual space if necessary at least $U_{1}$ has small dimension. Let $U_{j_{0}}$ be the largest $U_{i}$ of small dimension, and $U_{j_{1}}$ be the least $U_{i}$ with large dimension or $V$ otherwise. Choose a basis $e_{1}, \ldots, e_{n}$ of $V$ such that $U_{j_{0}}=\left\langle e_{1}, \ldots, e_{l}\right\rangle$ and $U_{j_{1}}=\left\langle e_{1}, \ldots, e_{k}\right\rangle$ for some $k, l$. The rest should be clear.

5.3. Analysis of a counterexample to Lemma $\mathrm{B}$ in which $s_{t}(\mathcal{M})$ is least possible. As mentioned above the alleged counterexample is really a triple $\langle r, s, \mathbf{M}\rangle$, but we focus on a typical member $\mathcal{M}$ of $\mathbf{M}$. We suppose $\langle r, s, \mathbf{M}\rangle$ has been chosen to minimize $s$. As usual $\mathcal{M}$ is $(M ; G)$.

The analysis will proceed as follows.

Step 1. $\mathcal{M}$ is primitive and $\operatorname{Soc}(G)$ is an elementary abelian $p$-group $A$. Choosing $b \in M$ we can identify $M$ with $A$ as in Lemma 10 and write $G=A H$, where $A$ acts by translation and $H=G_{b}$ acts by conjugation by the inverse.

Step 2. Look at $A$ as a vector space over $F_{p}$. It will be shown that $H$ is a primitive linear group and that, for any $N \unlhd H$, either $N$ acts irreducibly on $A$ or $|N|$ is bounded. Further, $H$ has a unique maximal normal abelian subgroup $B$ with bounded order. Let $H_{0}={ }_{\mathrm{dfn}} C_{H}(B)$.

Step 3. $H_{0} / B$ has no normal abelian subgroup.

Step 4. Let $S_{1} \leq H_{0}$ be a quasi-simple group projecting onto a simple factor of $\operatorname{Soc}\left(H_{0} / B\right)$. Let $S_{1}, \ldots, S_{l}$ be all the conjugates of $S_{1}$ in $H$. Then

$$
K=\prod\left\{S_{i}: 1 \leq i \leq l\right\} \unlhd H
$$

is a commuting product. It is shown that $l \leq 2$ and that $|H: K|$ is bounded.

Step 5 . Let $A_{1} \leq A$ be an irreducible $S_{1}$-module, and $\mathcal{M}_{1}=\left(A_{1} ; S_{1}\right)$. Then $S_{1}$ acts faithfully, so $\left|\bar{A}_{1}\right|$ is unbounded. Also, $s_{4}\left(\mathcal{M}_{1}\right)$ is bounded.

Thus we end up with a counterexample to Lemma 20. Below we give the details of the argument. In this section we allow more abuse of our notation for permutation structures than heretofore.

LEMMA 21. $\mathcal{M}$ is primitive.

ProOF. Suppose on the contrary that $E$ is a non-trivial $G$-invariant equivalence relation on $M$, i.e., suppose for infinitely many $\mathcal{M} \in \mathbf{M}$ such $E$ exists. Let $C$ be an $E$-class $C$ of maximum size seen as a permutation structure. Note that $s_{5}(C), s_{5}(\mathcal{M} / E)<s$. If $|C|$ is unbounded, some subset of the C's forms a new counterexample with $r$ unchanged. Otherwise, if $|C|<c<\omega$ as $\mathcal{M}$ runs through $M$, by Lemma 16 some subset of the $M / E$ 's forms a new counterexample with $r$ replaced by $\max (r, 6, c) \cdot\left(\log _{2} c+1\right)$. In either case the choice of $s$ is contradicted.

LEMMA 22. $\operatorname{Soc}(G)$ is abelian.

ProOF. Suppose on the contrary that $N=\operatorname{Soc}(G)$ is nonabelian for infinitely many $\mathcal{M} \in \mathbf{M}$. By Lemma 9 we can suppose that $N$ is simple. By Lemma 11, 
$N$ is either an alternating group or a classical group of large rank over $F_{q}$ with $q$ bounded in terms of $s_{4}(\mathcal{M})$ by Lemma 13. In Lemma 13 are enumerated the possibilities for $\mathcal{M}$ when $N$ is classical. In each case by inspection there are large sets of indiscernibles. From Lemma 14 the same is true when $N$ is alternating. Thus we have the desired contradiction.

As we go along we discard any $\mathcal{M}$ 's in $\mathbf{M}$ which do not have the properties being shown to hold for almost all $\mathcal{M} \in \mathbf{M}$. Now we write $A=\operatorname{Soc}(G)$. Choosing $b \in M$ we identify $M$ with $A$ (by matching $b$ with $0 \in A$ ). Denote $G_{b}$ by $H$. Then $A \cap H=(0)$ and $G=A H$. Set $\Phi=F_{p}$, where $A$ is an elementary abelian $p$-group. This last notation will be modified later.

LEMMA 23. Suppose $A_{1}<A$ and $A_{1}^{h} \cap A_{1}$ is $A_{1}$ or $(0)$ for all $h \in H$. Let $\mathcal{M}_{1}=$ $\left(A_{1} ; G_{1}\right)$ where $G_{1} \leq \operatorname{Sym}\left(A_{1}\right)$ is induced by $G\left(\left\{A_{1}\right\}\right)$. Then $s_{5}\left(\mathcal{M}_{1}\right)<s_{5}(\mathcal{M})$.

PROOF. We make two claims:

(i) If $g \in G$ and $\left|A_{1} \cap A_{1}^{g}\right|>1$, then $A_{1}=A_{1}^{g}$.

(ii) There exist distinct $a_{i} \in A(1 \leq i \leq 3)$ such that $\left(a_{1}, a_{2}, a_{3}\right)^{G} \cap A_{1}^{3}=\varnothing$.

To prove (i), compose $g$ with a translation in $A_{1}$, reducing to the case $g \in H$. For (ii) choose $a_{1}, a_{2} \in A_{1}$ with $a_{1} \neq a_{2}$ and $a_{3}$ in $A \backslash A_{1}$. Now apply (i). The two claims clearly suffice.

COROllaRY. As $\mathcal{M}$ runs through $\mathbf{M}$ there is a uniform finite bound on the cardinality of such $A_{1}$.

LEMMA 24. $H$ is primitive as a linear group over $\Phi=F_{p}$.

Proof. Towards a contradiction suppose $A=\bigoplus \sum\left\{A_{i} \in I\right\}$, where $(0)<A_{i}<$ $A(i \in I)$ and $H$ permutes the $A_{i}$. Then $E=\bigcup\left\{\left(A_{i} \backslash\{0\}\right)^{2}: i \in I\right\}$ is an equivalence relation on $C=\bigcup\left\{A_{i} \backslash\{0\}: i \in I\right\}$ invariant under $H$. From the last corollary, $\left|A_{i}\right|$ is bounded $(i \in I)$. Since $|H|$ is unbounded so is $|I|$. For $a=\sum\left\{a_{i}: i \in I\right\}$ with $a_{i} \in A_{i}(i \in I)$, define $n(a)=\left|\left\{i \in I: a_{i} \neq 0\right\}\right|$. Then $n: M \rightarrow \omega$ is a nonempty invariant under $H=G_{b}$ which takes $|I|+1$ different values. Hence $s_{2}(\mathcal{M})$ is unbounded, a contradiction.

LEMMA 25. If $N \unlhd H$, then either $N$ is irreducible on $A$ or $|N|$ is bounded.

Proof. Suppose $A_{1}<A$ is an irreducible $N$-module. By Clifford's Theorem $A$ is a direct sum of conjugates of $A_{1}$, and the copies of $A_{1}$ are isomorphic $N$-modules since $H$ is primitive. Thus $N$ acts faithfully on $A_{1}$. If $h \in H$, then $A_{1} \cap A_{1}^{h}=(0)$ or $A_{1}$ since $N^{h}=N$ and $A_{1}$ is irreducible for $N$. Hence $\left|A_{1}\right|$ is bounded by the corollary to Lemma 23 and so $|N|$ is also bounded.

COROLlaRY. If $N \unlhd H$ is nonabelian, then $\left|C_{H}(N)\right|$ is bounded.

Proof. If $C_{H}(N)$ acts irreducibily on $A$, then by Schur's Lemma and Wedderburn's Theorem the endomorphisms of $A$ which centralize $C_{H}(N)$ form a field. Thus $N$ is a subgroup of the multiplicative group of a field and hence abelian. So if $N$ is nonabelian, then $C_{H}(N)$ cannot act irreducibly on $A$, whence $\left|C_{H}(N)\right|$ is bounded by the lemma.

LEMMA 26. $H$ has a unique maximal abelian normal subgroup $B$, which is cyclic and of bounded order. 
ProOF. Let $B \unlhd H$ be maximal abelian. Towards a contradiction suppose first that $B$ acts irreducibly on $A$. By Schur's Lemma and Wedderburn's Theorem $C_{\text {End }(A)}(B)$ is a field $\Phi^{\prime}$ containing $B$. Let $a \in A \backslash\{0\}$; then $\left\{\varphi(a): \varphi \in \Phi^{\prime}\right\}$ is a $B$ module and so $\Phi^{\prime}$ can be identified with $A$ by $\varphi \mapsto \varphi(a)$. Under this identification $H_{a}$ acts as a subgroup of $\operatorname{Aut}\left(\Phi^{\prime}\right)$. As $|M| \rightarrow \infty$, so does $\left|\Phi^{\prime}\right|$, hence so do $s_{1}\left(\Phi^{\prime}\right)$ and $s_{3}(\mathcal{M})$. This contradicts the bound on $s_{5}(\mathcal{M})$. So $B$ cannot act irreducibly on $A$. By Lemma $25|B|$ is bounded.

Let $A_{1}<A$ be $B$-irreducible. As in Lemma 25 from Clifford's Theorem $B$ is faithful on $A_{1}$. By Schur's Lemma and Wedderburn's Theorem $C_{\operatorname{End}\left(A_{1}\right)}(B)$ is a field containing $B$. Hence $B$ is cyclic.

If $B_{1}, B_{2}$ are two possibilities for $B$, then $|H|$ and therefore $|M|$ can be bounded in terms of $\left|B_{1} B_{2}\right|$ and $\left|C_{H}\left(B_{1} B_{2}\right)\right|$ since $H / C_{H}\left(B_{1} B_{2}\right)$ is naturally embedded in $\operatorname{Aut}\left(B_{1} B_{2}\right)$. Since $|M| \rightarrow \infty$ while $\left|B_{1} B_{2}\right|$ is bounded, $\left|C_{H}\left(B_{1} B_{2}\right)\right| \rightarrow \infty$ and so by the last corollary $B_{1} B_{2}$ is abelian. Thus $B$ is unique.

We have completed Steps 1 and 2.

Let $C_{H}(B)$ be denoted $H_{0} .\left|H: H_{0}\right| \leq|\operatorname{Aut}(B)|$ is bounded. Therefore $H_{0}$ acts irreducibly on $A$ by Lemma 25 . Let $\Phi$ henceforth denote the field $C_{\operatorname{End}(A)}\left(H_{0}\right) \geq B$. Obviously $\Phi$ has the same characteristic $p$ as $A$. For every $\lambda \in \Phi \backslash\{0\}$ the map $x \mapsto \lambda x(x \in A)$ is $H_{0}$-invariant. Hence

$$
|\Phi|-2 \leq s_{2}\left(A \backslash\{0\} ; H_{0}\right) \leq\left|H: H_{0}\right| s_{2}(A \backslash\{0\} ; H) \leq\left|H: H_{0}\right| s_{3}(\mathcal{M}) .
$$

Thus $|\Phi|$ is bounded. Let $k=\operatorname{dim}_{\Phi}(A)$. From Lemma $26 B=Z\left(H_{0}\right)$.

LEMMA 27. $H_{0} / B$ has no normal abelian subgroup $>(1)$.

Proof. Towards a contradiction suppose $B<F \unlhd H_{0}$ with $F / B$ abelian. By choosing $F$ minimal and then replacing $F$ by the subgroup generated by $F$ and its conjugates in $H$ we can suppose $F \unlhd H$. By the corollary to Lemma $25,\left|C_{H}(F)\right|$ is bounded. Since $|H| \leq\left|C_{H}(F)\right||\operatorname{Aut}(F)|,|F|$ is not bounded, whence $\left|C_{H}\left(C_{H}(F)\right)\right|$ is not bounded, whence $C_{H}(F)$ is abelian. Thus $C_{H}(F)=B$. By [10, Theorem 2], we have $C_{H_{0}}(F / B)=F$. By $\left[22\right.$, Lemma 10] $|F / B| \leq k^{2}$. For an abelian group $P,|\operatorname{Aut}(P)| \leq|P|^{\log _{2}|P|}$. Since $H_{0} / F$ acts faithfully on $F / B$,

$$
\left|H_{0} / F\right| \leq|\operatorname{Aut}(F / B)| \leq k^{4 \log _{2} k}
$$

and so

$$
|H| \leq\left|H: H_{0}\right|\left|H_{0}: F\right||F: B||B| \leq|B||\operatorname{Aut}(B)| k^{2+4 \log _{2} k} .
$$

On the other hand $|A \backslash\{0\}|=|\Phi|^{k}-1 \geq 2^{k}-1$. As $|M| \rightarrow \infty$, so does $k$, and hence so does $|A \backslash\{0\}| /|H|$. Therefore $s_{2}(\mathcal{M})=s_{1}(A \backslash\{0\} ; H) \rightarrow \infty$ as $|M| \rightarrow \infty$ which contradicts the bound on $s_{5}(\mathcal{M})$.

The last lemma is patterned on Lemma 3.1 of [3]. This completes Step 3 of our analysis.

Recall that a group $S$ is called quasi-simple if $S^{\prime}=S$ and $S / Z(S)$ is simple. Let $S_{1} \leq H_{0}$ be a quasi-simple group projecting onto a simple factor $T_{1}$ of $\operatorname{Soc}\left(H_{0} / B\right)$. Notice that any two preimages of $T_{1}$ have the same derived group. Thus $S_{1}$ is the unique minimal preimage of $T_{1}$. Let $S_{1}, \ldots, S_{l}$ be all the conjugates of $S_{1}$ in $H$, and $T_{1}, \ldots, T_{l}$ be the corresponding conjugates of $T_{1}$ in $H / B$. The $T_{i}$ are minimal normal subgroups of $\operatorname{Soc}(H / B)$ and so centralize each other. Hence the $S_{i}$ normalize each other. Consider the isomorphism $s \mapsto s^{t}\left(s \in S_{i}\right)$, where $t \in S_{j}$ 
for some $j \neq i$. Since $T_{i}, T_{j}$ commute, $s^{t}=s z_{s}$ for some $z_{s} \in B \cap S_{i}$. If $s$ is a commutator, $s^{t}$ is clearly $s$. But $S_{i}=S_{i}^{\prime}$, whence $s^{t}=s\left(s \in S_{i}\right)$, i.e., $S_{i}, S_{j}$ centralize each other. Let $K=\prod\left\{S_{i}: 1 \leq i \leq l\right\}$. Clearly $K \unlhd H$.

LEMMA 28. Either $\left|T_{1}\right|$ is bounded or $T_{1}$ is an alternating group or a classical group over $F_{q}$ with $q$ bounded. In particular, $\left|\operatorname{Aut}\left(T_{1}\right) / T_{1}\right|$ is bounded.

ProOF. Suppose that $\left|T_{1}\right|$ is unbounded. Since $B \cap K \triangleleft H$, the orbits of $B \cap K$ on $A$ are the classes of an $H$-invariant equivalence relation $E_{B \cap K}$. Similarly for the orbits of $K$. Since $|B|$ is bounded so is the size of the orbits of $B \cap K$. Since $|K|$ is unbounded so is the size of the orbits of $K$ by Lemma 25. Therefore we can choose a $K$-orbit $Q$, and a maximal $H(\{Q\})$-invariant equivalence relation $E$ on $Q$ refined by $E_{B \cap K}$ such that $R=Q / E$ is not a singleton. Let $H_{1}, K_{1} \leq \operatorname{Sym}(R)$ be the groups induced by $H(\{Q\})$ and $K=K(\{Q\})$. Let $R=\left(R ; H_{1}\right)$. Then $R$ is primitive and $(1)<K_{1} \unlhd H_{1}$. Since $Q$ is a class of a $\{b\}$-definable equivalence relation, $s_{4}(R) \leq s_{5}(\mathcal{M})$. Since we arranged for the action of $B \cap K$ to be trivial and for that of $K$ to be nontrivial on $R$, $\operatorname{Soc}\left(H_{1}\right)$ has a direct factor isomorphic to $T_{1}$. Applying Lemmas 9, 11, and 13 we conclude that either $T_{1}$ is an alternating group, or $T_{1}$ is a classical simple group over $F_{q}$ with $q$ bounded.

For the rest note that $|\operatorname{Aut}(T) / T|=2$ for an alternating group and is bounded in terms of $q$ for a classical simple group.

LEMMA 29. $l \leq 2$ and $|H: K|$ is bounded.

PROOF. Let $d$ be the least degree of an irreducible linear representation of $S_{1}$. From [3, Proof of 2.9], since $A$ provides an irreducible linear representation of $K=\prod\left\{S_{i}: 1 \leq i \leq l\right\}$ over $F_{p}$, the dimension of $A$ as a vector space over $F_{p}$ is at least $d^{l}$. Thus $|A| \geq p^{d^{l}} \geq 2^{d^{i}}$.

Since $K=K^{\prime}$ the natural homomorphism from $\operatorname{Aut}(K)$ into $\operatorname{Aut}(K / K \cap B)$ is an embedding. Therefore

$$
|\operatorname{Aut}(K)| \leq\left|\operatorname{Aut}\left(T_{1} \times \cdots \times T_{l}\right)\right|=l !\left|\operatorname{Aut}\left(T_{1}\right)\right|^{l} .
$$

Also

$$
\begin{aligned}
|H: K| & \leq\left|C_{H}(K)\right||\operatorname{Aut}(K)| /|K| \leq\left|C_{H}(K)\right|\left|\operatorname{Aut}\left(T_{1}\right) / T_{1}\right|^{l} l ! \\
& <\left|C_{H}(K)\right|\left|\operatorname{Aut}\left(T_{1}\right) / T_{1}\right|^{l} 3^{l^{2}} .
\end{aligned}
$$

From the corollary to Lemma $25\left|C_{H}(K)\right|$ is bounded, and from the last lemma $\left|\operatorname{Aut}\left(T_{1}\right) / T_{1}\right|$ is bounded. Choose a constant $C$ bounding $\left|C_{H}(N)\right|,\left|\operatorname{Aut}\left(T_{1}\right) / T_{1}\right|$, 3 , and also $q$ if $T_{1}$ is a classical group. Since $H$ has at most $s_{2}(\mathcal{M})<s$ orbits on $A \backslash\{0\}, K$ has at most $s|H: K| \leq s C^{1+l+l^{2}}$ orbits on $A \backslash\{0\}$. Each $K$-orbit has size $\leq|K| \leq\left|S_{1}\right|^{l}$. Therefore

$$
2^{d^{l}}-1 \leq|A \backslash\{0\}| \leq s C^{1+l+l^{2}}\left|S_{1}\right|^{l}
$$

If we suppose that $\left|S_{1}\right|$ is bounded, we can suppose $\left|S_{1}\right| \leq C$ so that $2^{d^{l}}-l \leq$ $s C^{1+2 l+l^{2}}$. Since $S_{1}$ is nonabelian $d \geq 2$. Thus $l$ is bounded. By (\#) $|A|$ is bounded, a contradiction. Thus $\left|S_{1}\right|$ and hence $\left|T_{i}\right|$ are unbounded.

By Lemma 28, $T_{1}$ is either $\operatorname{Alt}(n)$ or a classical group $P G(V)$ over $F_{q}$ where $q$ is bounded and $\operatorname{dim}(V)=n$. Thus $C$ may be chosen so that $\left|S_{1}\right| \leq C^{n^{2}}$. From Lemma $17 d \geq n / 30$. Now (\#) yields

$$
2^{(n / 30)^{l}}-1 \leq s C^{1+l+l^{2}+l n^{2}} \text {. }
$$


Hence $l \leq 2$ since $n \rightarrow \infty$ as $|M| \rightarrow \infty$. Once $l$ is bounded, the bound on $|H: K|$ is clear.

Let $A_{1} \leq A$ be an irreducible $S_{1}$-module and $\mathcal{M}_{1}=\left(A_{1} ; G_{1}\right)$ where $G_{1}$ is induced by $S_{1}$.

LEMMA 30. $\left|M_{1}\right|$ is unbounded, $S_{1}$ acts faithfully on $A_{1}$, and $s_{4}\left(\mathcal{M}_{1}\right)$ is bounded.

PROOF. We shall assume $l=2$ because if $l=1$ the conclusions are already clear since $K$ is irreducible on $A, s_{4}(A ; H)<s_{5}(\mathcal{M})$, and $|H: K|$ is bounded.

Applying Clifford's Theorem to $K, A$ is a direct sum of $S_{1}$-modules of the form $A_{1}^{g}\left(g \in S_{2}\right)$. Since $\left[S_{1}, S_{2}\right]=1$, these $S_{1}$-modules are pairwise isomorphic. Thus $S_{1}$ acts faithfully on $A_{1}$. Hence $\left|M_{1}\right|$ is unbounded since $\left|S_{1}\right|$ is. $A_{1} \backslash\{0\}$ is an equivalence class of a $K$-invariant equivalence relation on $A$. Let $K_{1} \leq \operatorname{Sym}\left(A_{1}\right)$ be the group induced by $K$. Then $s_{4}\left(A_{1} ; K_{1}\right) \leq s_{4}(A ; K)$. Let $\Phi_{1}=C_{\operatorname{End}\left(A_{1}\right)}\left(S_{1}\right)$. By Schur's Lemma and Wedderburn's Theorem, $\Phi_{1}$ is a field. Now $K\left(\left\{A_{1}\right\}\right)=S_{1} T$, a commuting product. Thus $K_{1}=S_{1} T_{1}$, a commuting product. We have $T_{1} \leq \Phi_{1}$ and, for $\lambda \in \Phi_{1}, x \mapsto \lambda x\left(x \in A_{1}\right)$ is a $K_{1}$-invariant map. Therefore

$$
\begin{aligned}
\left|T_{1}\right| & \leq\left|\Phi_{1}\right| \leq s_{2}\left(A_{1} ; K_{1}\right) \leq s_{4}\left(A_{1} ; K_{1}\right) \leq s_{4}(A ; K) \\
& \leq|H: K| s_{4}(A ; H)=|H: K| s_{5}(\mathcal{M}) .
\end{aligned}
$$

It follows that $\left|K_{1}: S_{1}\right|$ is bounded and hence that $s_{4}\left(A_{1} ; S_{1}\right)=s_{4}\left(\mathcal{M}_{1}\right)$ is also bounded.

This completes Step 5 of our plan and the proof of Lemma B.

\section{REFERENCES}

1. E. Artin, Geometric algebra, Interscience, New York, 1966.

2. M. Aschbacher and L. Scott, Maximal subgroups of finite groups, J. Algebra 92 (1985), 44-80.

3. L. Babai, P. Cameron and P. Palfy, On the orders of primitive groups with restricted nonabelian composition factors, J. Algebra 79 (1982), 161-168.

4. E. Bannai, Maximal subgroups of low rank of finite symmetric and alternating groups, J. Fac. Sci. Univ. Tokyo Sect. I 18 (1971/2), 475-486.

5. P. Cameron, Finite permutation groups and finite simple groups, Bull. London Math. Soc. 13 (1981), 1-22.

6. R. W. Carter, Simple groups of Lie type, Wiley, New York, 1972.

7. G. Cherlin, L. Harrington and A. H. Lachlan, $\aleph_{0}$-categorical, $\aleph_{0}$-stable structures, Ann. Pure Appl. Logic 28 (1985), 103-135.

8. C. W. Curtis, W. M. Kantor and G. M. Seitz, The 2-transitive representations of Chevalley groups, Trans. Amer. Math. Soc. 218 (1976), 1-57.

9. D. Dieudonné, La géométrie des groupes classiques, Springer-Verlag, Berlin, 1963.

10. I. M. Isaacs, Character degrees and derived length of a solvable group, Canad. J. Math. 27 (1975), 146-151.

11. W. M. Kantor, Permutation representations of the finite classical groups of small degree or rank, J. Algebra 60 (1979), 158-168.

12. A. H. Lachlan, Countable homogeneous tournaments, Trans. Amer. Math. Soc. 284 (1984), 231-261.

13. __ On countable stable structures which are homogeneous for a finite relational language, Israel J. Math. 49 (1984), 69-153.

14. A. H. Lachlan and S. Shelah, Stable structures homogeneous for a finite binary language, Israel J. Math. 49 (1984), 155-180.

15. A. H. Lachlan and R. E. Woodrow, Countable ultrahomogeneous graphs, Trans. Amer. Math. Soc. 262 (1980), 51-94. 
16. J. Landazuri and G. M. Seitz, J. Algebra 32 (1974), 418-443.

17. D. Passman, Permutation groups, Benjamin, New York, 1968.

18. G. M. Seitz, Flag-transitive subgroups of Chevalley groups, Ann. of Math. (2) 97 (1973), 27-56.

19. , Small rank permutation representations of finite Chevalley groups, J. Algebra 28 (1974), 508-517.

20. S. Shelah, Classification theory, North-Holland, Amsterdam, 1978.

21. D. Stanton, Some q-Krawtchouk polynomials on Chevalley groups, Amer. J. Math. 102 (1980), 625-662.

22. D. Suprunenko, Soluble and nilpotent linear groups, Transl. Math. Monographs., vol. 9, Amer. Math. Soc., Providence, R.I., 1963.

Department of Mathematics, Rutgers University, New Brunswick, NeW JerSEY 08903

DePARTMENT OF MATHEMATICS, Simon FRASER UNiVERSity, BuRNABy V5A 1 S6 B.C., CANADA 